Florida International University FIU Digital Commons

\title{
Extended Coverage for Public Safety and Critical Communications Using Multi-hop and D2D Communications
}

Leonardo Babun

lbabu002@fiu.edu

DOI: $10.25148 /$ etd.FI15032110

Follow this and additional works at: https://digitalcommons.fiu.edu/etd

Part of the Systems and Communications Commons

\section{Recommended Citation}

Babun, Leonardo, "Extended Coverage for Public Safety and Critical Communications Using Multi-hop and D2D Communications" (2015). FIU Electronic Theses and Dissertations. 1920.

https://digitalcommons.fiu.edu/etd/1920 


\title{
FLORIDA INTERNATIONAL UNIVERSITY \\ Miami, Florida
}

EXTENDED COVERAGE FOR PUBLIC SAFETY AND CRITICAL COMMUNICATIONS USING MULTI-HOP AND D2D COMMUNICATIONS

A thesis submitted in partial fulfillment of the

\author{
requirements for the degree of \\ MASTER OF SCIENCE \\ in \\ ELECTRICAL ENGINEERING \\ by \\ Leonardo Babun
}


To: Dean Amir Mirmiran

College of Engineering and Computing

This thesis, written by Leonardo Babun, and entitled Extended Coverage for Public Safety and Critical Communications using Multi-hop and D2D Communications, having been approved in respect to style and intellectual content, is referred to you for judgment.

We have read this thesis and recommend that it be approved.

Shaolei Ren

Neiyer Correal

Nezih Pala

Ismail Guvenc, Major Professor

Date of Defense: March 26, 2015

The thesis of Leonardo Babun is approved.

$\begin{array}{r}\text { Dean Amir Mirmiran } \\ \text { College of Engineering and Computing } \\ \hline \begin{array}{r}\text { Dean Lakshmi N. Reddi } \\ \text { University Graduate School }\end{array}\end{array}$

Florida International University, 2015 
(c) Copyright 2015 by Leonardo Babun

All rights reserved. 


\section{DEDICATION}

To my family. 


\section{ACKNOWLEDGMENTS}

I would like to express my sincere gratitude to my major professor, Dr. Ismail Guvenc, for his mentorship, motivation and patience throughout my graduate studies. His insights and words of encouragement have often inspired and encouraged me to overcome all difficulties. I am deeply indebted to him for his tireless support.

I also extend my thanks to my committee members, Dr. Shaolei Ren, Dr. Neiyer Correal and Dr. Nezih Pala for their kind suggestions, efforts, and contributions to this work.

I am also grateful to the Mobile, Pervasive, and Autonomous Communications Technologies (MPACT) group, whose members have become real supportive friends during all my graduate studies, to the Department of Electrical and Computer Engineering, and to FIU for providing me with this research and study opportunity.

Special thanks to Med-Tek LLC, where I work as a full time engineer, for their support and understanding during all my graduate studies.

Finally, I would like to thank my family for their love and patience. 


\section{ABSTRACT OF THE THESIS \\ EXTENDED COVERAGE FOR PUBLIC SAFETY AND CRITICAL COMMUNICATIONS USING MULTI-HOP AND D2D COMMUNICATIONS}

by

Leonardo Babun

Florida International University, 2015

Miami, Florida

Professor Ismail Guvenc, Major Professor

In this thesis, we proposed the use of device-to-device (D2D) communications for extending the coverage area of active base stations, for public safety communications with partial coverage. A 3GPP standard compliant D2D system level simulator is developed for HetNets and public safety scenarios and used to evaluate the performance of D2D discovery and communications underlying cellular networks.

For D2D discovery, the benefits of time-domain inter-cell interference coordination (ICIC) approaches by using almost blank subframes were evaluated. Also, the use of multi-hop is proposed to improve, even further, the performance of the D2D discovery process.

Finally, the possibility of using multi-hop D2D communications for extending the coverage area of active base stations was evaluated. Improvements in energy and spectral efficiency, when compared with the case of direct UE-eNB communications, were demonstrated. Moreover, UE power control techniques were applied to reduce the effects of interference from neighboring D2D links. 


\section{TABLE OF CONTENTS}

1. INTRODUCTION . . . . . . . . . . . . . . . . . . 1

1.1 Requirements and Services of Public Safety Networks . . . . . . . . . . 2

1.2 Long Term Evolution and Public Safety . . . . . . . . . . . . . . 4

1.3 Problem Statement . . . . . . . . . . . . . . . . . . 7

1.4 Research Objectives . . . . . . . . . . . . . . . . . . 8

1.5 Organization of the Thesis . . . . . . . . . . . . . . . . 9

2. OVERVIEW OF 3GPP D2D COMMUNICATIONS . . . . . . . . . 11

2.1 Introduction to D2D Proximity Services . . . . . . . . . . . . . . 12

2.1 .1 D2D Key Functions . . . . . . . . . . . . . . . . . . . 13

2.2 3GPP D2D Standardization . . . . . . . . . . . . . . . . 14

2.3 Long Term Evolution D2D Communications Resource Use . . . . . . . 18

2.4 3GPP D2D Evaluation and Channel Models . . . . . . . . . . . . . . . . 19

2.4.1 3GPP UE Dropping in D2D Scenarios . . . . . . . . . . . . . . 21

2.4.2 3GPP Device-to-Device Evaluation Metrics . . . . . . . . . . . 21

2.4.3 3GPP D2D Channel Propagation Model . . . . . . . . . . . . . . . 22

3. D2D DISCOVERY AND INTER-CELL INTERFERENCE COORDINATION

IN LTE-A HETEROGENEOUS NETWORKS . . . . . . . . . . . . . . 24

3.1 Introduction to D2D Discovery Process . . . . . . . . . . . . . . . 24

3.2 D2D System Level Simulation Environment . . . . . . . . . . . . 27

3.2 .1 Initialization . . . . . . . . . . . . . . . . . . . . . 29

3.2 .2 Channel Modeling . . . . . . . . . . . . . . . . . . . . . . . . . . 29

3.2 .3 Resource Group Selection . . . . . . . . . . . . . . . . . . . . 30

3.2.4 Signal-to-Interference-plus-Noise-ratio Calculation . . . . . . . . . . . . 31

3.2 .5 D2D Discovery algorithms . . . . . . . . . . . . . . . 31

3.2 .6 Mobility . . . . . . . . . . . . . . . . . . . . . 31

3.2 .7 Evaluation . . . . . . . . . . . . . . . . . . . . 31

3.3 D2D Interference Coordination $\ldots \ldots \ldots \ldots \ldots \ldots \ldots$

3.4 D2D Discovery Algorithms . . . . . . . . . . . . . . . . . 33

3.5 System Model Simulation Results on D2D Discovery . . . . . . . . 37

4. D2D COMMUNICATIONS IN PUBLIC SAFETY PARTIAL COVERAGE

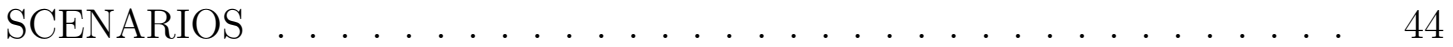

4.1 Introduction to Device-to-Device Communication Process . . . . . . . . . 44

4.2 D2D Communications Routing Algorithms . . . . . . . . . . . . 45

4.3 D2D System Level Simulation Environment for Partial Coverage Scenarios 48

4.3.1 Energy Efficiency and Spectral Efficiency for Multi-hop D2D Communications . . . . . . . . . . . . . . . 51

4.4 System Model Simulation Results on D2D Communications . . . . . . 52 


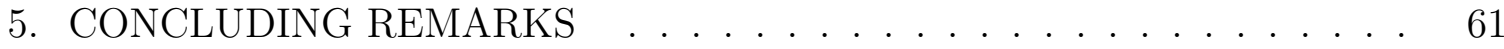

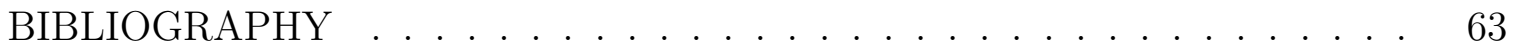




\section{LIST OF TABLES}

TABLE

PAGE

2.1 Potential applications of D2D based on mobile user proximity. . . . . . 15

2.2 ProSe use cases and scenarios defined in TR $22.803[1] \ldots \ldots \ldots \ldots$. . 17

2.3 Channel model assumptions for simulating D2D scenarios. . . . . . . . 23

3.1 Simulation parameters for D2D discovery. . . . . . . . . . . 38

4.1 Simulation parameters for D2D communications. . . . . . . . . . . 49 


\section{LIST OF FIGURES}

FIGURE

PAGE

1.1 Extended coverage using D2D and multi-hop in public safety communications scenario with partial coverage. . . . . . . . . . .

2.1 D2D communications as an underlay to a cellular network for public safety: a) locally routed D2D communications, b) D2D communication without network support, c) LTE communication path for UEs in MBS coverage, d) UE to network relay for out-of coverage UE in public safety scenario, e) D2D direct communication in public safety scenario. .

2.2 D2D standarization roadmap [2]. . . . . . . . . . . . . . . . 16

3.1 Layout option 5 according to R1-131755 overlaid by small cell BSs. . . . 25

3.2 D2D system level simulator flowchart. . . . . . . . . . . . . . . 28

3.3 (a) Frame structure for different ABS rates $\left(R_{\mathrm{ABS}}\right)$, (b) DR distribution over $N_{\mathrm{T}} \times N_{\mathrm{F}}$ LTE-A subframe. . . . . . . . . . .

3.4 Illustration of multi-hop discovery of far away UEs. . . . . . . . . . . . . 36

3.5 CDF of number of discovered UEs in the absence of ICIC for random and smart PDRID selection, and $\gamma_{\text {thresh }}=8 \mathrm{~dB} \ldots \ldots \ldots$

3.6 Average number of discovered UEs vs $N_{T}$ for various ABS ratios in case of macro ICIC and $\gamma_{\text {thresh }}=8 \mathrm{~dB} . \ldots \ldots \ldots$

3.7 Average number of discovered UEs vs $N_{T}$ for various ABS ratios in case of pico ICIC and $\gamma_{\text {thresh }}=8 \mathrm{~dB} . \ldots \ldots$. . . . . . . .

3.8 Average number of discovered UEs vs $\gamma_{\text {thresh }}$ for various ABS ratios in case of macro ICIC. . . . . . . . . . . . . . . . . . . . .

3.9 Average number of discovered UEs vs $\gamma_{\text {thresh }}$ for various ABS ratios in case of pico ICIC. . . . . . . . . . . . . . . . . . . . .

3.10 Average number of discovered UEs for multi-hop in the absence of ICIC.

3.11 Average number of discovered UEs for multi-hop discovery and different

ABS ratios. . . . . . . . . . . . . . . .

4.1 Multi-hop routes model used for communication purposes in public safety partial coverage scenario. . . . . . . . . . . . .

4.2 Full multi-hop routes scheduling in LTE time frames for D2D communications received power $P_{r}(r, l, h)$ and interference received power $I_{r}(r, l, h) .50$

4.3 3GPP public safety partial coverage scenario [3] and defined D2D routes for evaluation purposes. . . . . . . . . . . . . . . 
4.4 Energy efficiency vs number of hops for maximum UE transmission power. 54

4.5 Energy efficiency vs spectral efficiency for maximum UE transmission power. 54

4.6 System model representation of $B S_{2}$ with $400 \mathrm{~m}$ location offset. . . . . . 55

4.7 Energy efficiency performance vs number of hops while reducing the UE transmission power. ................... 56

4.8 Energy efficiency performance vs spectral efficiency while reducing the UE transmission power. . . . . . . . . . . . . . . . 57

4.9 Spectral efficiency vs number of hops for different $B S_{2}$ position offsets. . 57

4.10 Energy efficiency vs number of hops for different $B S_{2}$ position offsets. . 58

4.11 Energy efficiency vs spectral efficiency for different $B S_{2}$ position offsets. $\quad 58$

4.12 Spectral efficiency performance vs number of hops in route 1 with and without power control for high interference scenario. . . . . . . . . 59

4.13 Energy efficiency performance vs spectral efficiency for D2D communications in route 1, with and without power control for high interference scenario. . . . . . . . . . . . . . . . . . 


\section{CHAPTER 1}

\section{INTRODUCTION}

Natural disasters or other emergency crisis are usually unplanned events that cause panic conditions in the civilian population and affect existing resources. Typically, public safety officers and responders include law enforcement, firefighters, emergency medical personnel, military organizations, volunteers groups, and other local and national organizations. The aim of these organizations and personnel is to protect people and assets, and respond as soon as possible to the disaster location, in order to provide medical attention and organize rescue operations.

Communications is an essential element in various operational scenarios and at different levels of the hierarchy of public safety. In this type of scenario, communication capabilities need to be provided in very challenging environments, where critical infrastructures (e.g. base stations) are often degraded or destroyed by the impact of the catastrophic event. Despite this, public safety responders should be able to exchange information in a timely manner to coordinate the relief efforts and to develop situational awareness. Timely information sharing and the development of shared situational awareness is critical.

Public safety communications play a critical role during disaster response and recovery. In large-scale natural disasters, many different public safety organizations may be involved with different information technology and communication systems. At the same time, the commercial communications infrastructure and resources that are still usable, have to be utilized to alert and communicate with the civilian population.

The ability of the public safety responder emergency services to communicate among themselves and to share multimedia information directly affects the ability to save lives. Although some first responders make use of WiFi in the interference-prone 
unlicensed spectrum, the majority of the first responders linger in a $2 \mathrm{G}$ world [4]. This represents a contradiction since public safety networks are seen as mission-critical; that is, reliable, resilient, and secure, while meeting other stringent functionality requirements in terms of service accessibility, radio coverage, end-to-end performance, and device characteristics [5].

\subsection{Requirements and Services of Public Safety Networks}

A public safety communication system provides the ways for public safety responders to accomplish their mission by communicating in a variety of media, foremost of which is speech. Public safety agents are typically organized in groups of individuals with different responsibilities. Each group would be led by an organizer or dispatcher with authority and supervisory responsibilities.

A group call involves the communications to all members of the group and the permission of speak is normally controlled by the supervisor. Sometimes, the members of the group will receive multiple calls simultaneously, but they will have to use their device to listen to the one with highest priority, previously assigned by the system. Also, responders may indicate emergencies causing all of their subsequent communications to the group to be treated as an emergency group call. Communication to group members is not exclusive to speech, as data messaging can also be sent. This data communication may involve any amount of data in any format, text, image, video etc [4].

In parallel with the complexity that involves the communication mechanisms during disasters, many public safety communications have a mission-critical aspect that places special requirements on the underlying radio technologies. When a public safety responder pushes the button on their devices to request to speak, they need the confidence that they will be successful with extremely high priority. In an emergency 
situation when congestion may exist, the emergency group call must go through or lives will be lost.

The current private and professional mobile radio technologies used for public safety organizations offer a rich set of voice-centralized services but have very limited data transmission capabilities. The diverse set of roles and scenarios of public safety requires various services and capabilities from communication systems. The SAFECOM program of the US Department of Homeland Security uses the following definitions [6]:

- Interactive voice communications: Among public safety officers.

- Non-interactive voice communications: Occurs when a dispatcher or supervisor alerts all members of the group about emergency situations or acts to share information, without an immediate response being required.

- Interactive data communications: When there is query made and a response provided.

- Non-interactive data communications: Among public safety officers.

There are more services that can be identified: voice, video, data connectivity, broadcast, multicast and/or push-to-talk. Depending on the scenario and the type of the emergency, several communication challenges can be present. For example, during emergencies in large urban areas, communication normally has to be done by using the existing local wireless communication networks, potentially connected to the public safety command. In this case, high buildings and other obstacles are likely to inhibit wireless communications. On the other hand, for crisis in large natural areas or when the local infrastructure is damaged or non-present, lack of coverage, traffic capacity, and traffic scalability represent major issues $[4,7]$.

There is a wide consensus among public safety organizations regarding the need of dedicated public safety networks for mission-critical communications because current 
commercial networks are not considered capable of providing the required degree of services availability, reliability and security. Nevertheless, the significant investment required to deploy dedicated public safety networks may not be considered affordable for some public administrations. Hence, while some countries can deploy dedicated public safety networks with nationwide coverage, others may decide to cover only some critical areas. Even when dedicated public safety networks can be rolled out, the unpredictable nature of the time, place and scale of an incident renders it virtually impossible to ensure that the public safety responders will have proper support from the public safety networks during the emergency, again, because dedicated networks are also vulnerable to lack of coverage, capacity or damaged infrastructure. In this context, significant opportunities for creating and exploiting alliances between public safety networks and commercial networks (e.g Long Term Evolution (LTE)) have been discussed, with the possibility of creating increased aggregated capacity, improved resiliency and enhanced radio coverage [5].

\section{$1.2 \quad$ Long Term Evolution and Public Safety}

LTE is being widely deployed as the global mobile broadband standard. Perhaps the main benefit in the use of LTE for public safety is having large-scale deployment of LTE, which allows less expensive equipment based on unified standards. These standards can be adopted by all public safety agencies and organizations globally, thus sharing scale with non-public safety applications of LTE.

Communication bearers, specially speech bearers, transported across LTE system require varying priorities and have multiple characteristics so, to accomplish the management of such diverse and dynamic bearer traffic, LTE provides Quality of Service (QoS) controls referred to as QoS class identity (QCI) and access retention priority (ARP). Currently, LTE standards support QoS controls for unicast bear- 
ers (single device). Also, a broadcast capability called evolved multimedia broadcast and multicast service (eMBMS) also exist in LTE and will be required to provide communications to large number of public safety responders in a close geographic region.

LTE system also offers great flexibility to support a wide variety of deployment scenarios and operator's needs, including:

- Homogeneous and heterogeneous networks: Macro, pico and femtocells.

- Single-carrier and multicarrier: Including cross-carrier scheduling.

- Support for both frequency-division duplex (FDD) and time-division duplex (TDD).

- Intercell coordination in time and frequency domains.

- Single and multipoint transmission and reception.

- Interworking and mobility with other radio access technologies: Such as code-division multiple access (CDMA) and wireless LAN (WLAN).

- Range of User Equipment (UE) categories and capabilities.

- Unicast and broadcast service support.

As we mentioned before, due to the critical nature of the different emergency communications, public safety requires that the network supports diverse traffic in a robust environment. Some of this robustness is provided by LTE through standardization; other aspects result from careful network planning. In general, robustness goes beyond just cell and capacity planning, it demands that alternative paths be available in the event of congestion and resources outages. When part of the public safety network fails, the remainder of the network must continue to provide services to the greatest extent possible. The LTE transport infrastructure must support such continued communications. The way to accomplish this will likely involve both application-level 
and LTE network level enhancements and it may incorporate what is known as direct communications.

Direct mode communications is the ability of two or more devices to communicate directly without the use of network infrastructure. This direct link would be used to connect two or more individuals when out-of-range of the network cell. In this direction, the Third Generation Partnership Project (3GPP) is working hard in defining requirements for such direct mode communications in LTE, and to develop solutions to support multiparty direct mode communications, e.g device-to-device (D2D) communications.

Therefore, LTE is well positioned to meet the requirements for the evolution of public safety systems. On the other hand, the adoption of LTE for public safety requires the specification of services, which are present in the current digital public safety wireless communication technologies but they are not usually defined in the commercial domain [8]. 3GPP has started the standardization activity in three main areas, which are related with the public safety domain:

1. Proximity services (ProSe): That identify mobiles in physical proximity and enable optimized communications between them (D2D communications) [9].

2. Group call system enablers: That support fundamental requirements for efficient and dynamic group communications operations such as one-to-many calling and dispatcher working [10].

\section{Public Safety Broadband High Power User Equipment for Band 14 for}

Region 2: This activity has the objective to specify high power user equipments in vehicular terminals [11].

All in all, the adoption of commercial mainstream LTE technology to satisfy public safety data communication needs is gaining momentum and offers significant opportunities to create and exploit the synergies between the commercial and public safety 
domains, which have remained almost entirely separate to date. LTE promises not only to provide an evolution path toward broadband capabilities for existing and new

public safety networks, but also opens new business opportunities for operators of commercial LTE networks.

\subsection{Problem Statement}

Because of their size and deployment characteristics, macro base stations (MBSs) are vulnerable to massive disaster events. During emergency situations, we should expect that only a reduced number of MBSs remain active due the direct impact of the disaster [12]. This is a typical case of partial coverage scenario [13].

In partial coverage scenarios, large metropolitan areas can lose cellular coverage when government agencies and public need it most. At this point, public safety and emergency communications become critical and guaranteeing communications with acceptable Quality of Services (QoS) is a big challenge. During large-scale emergencies, we should expect a large number of people trying to communicate at the same time, collapsing very easily the limited amount of available resources. LTE and D2D communications can be a good solution for extending the coverage of those MBSs that remain active in a partial coverage scenario [14-17]. By using multihop communication techniques, it is possible to connect the source (far-away UE) to the destination (MBS), with the intervention of other UEs, acting as temporary wireless relay (see Fig. 1.1) [18]. There are several multi-hop algorithms that can be applied [18-22], and selecting the correct routing technique is a key factor when using multi-hop since every technique carries different performances for Energy Efficiency (EE), Spectral Efficiency (SE), numbers of hops, delay etc.

In order to avoid causing intolerable interferences to cellular users, the D2D links use relatively small transmit power, so every D2D link is capable of covering small 


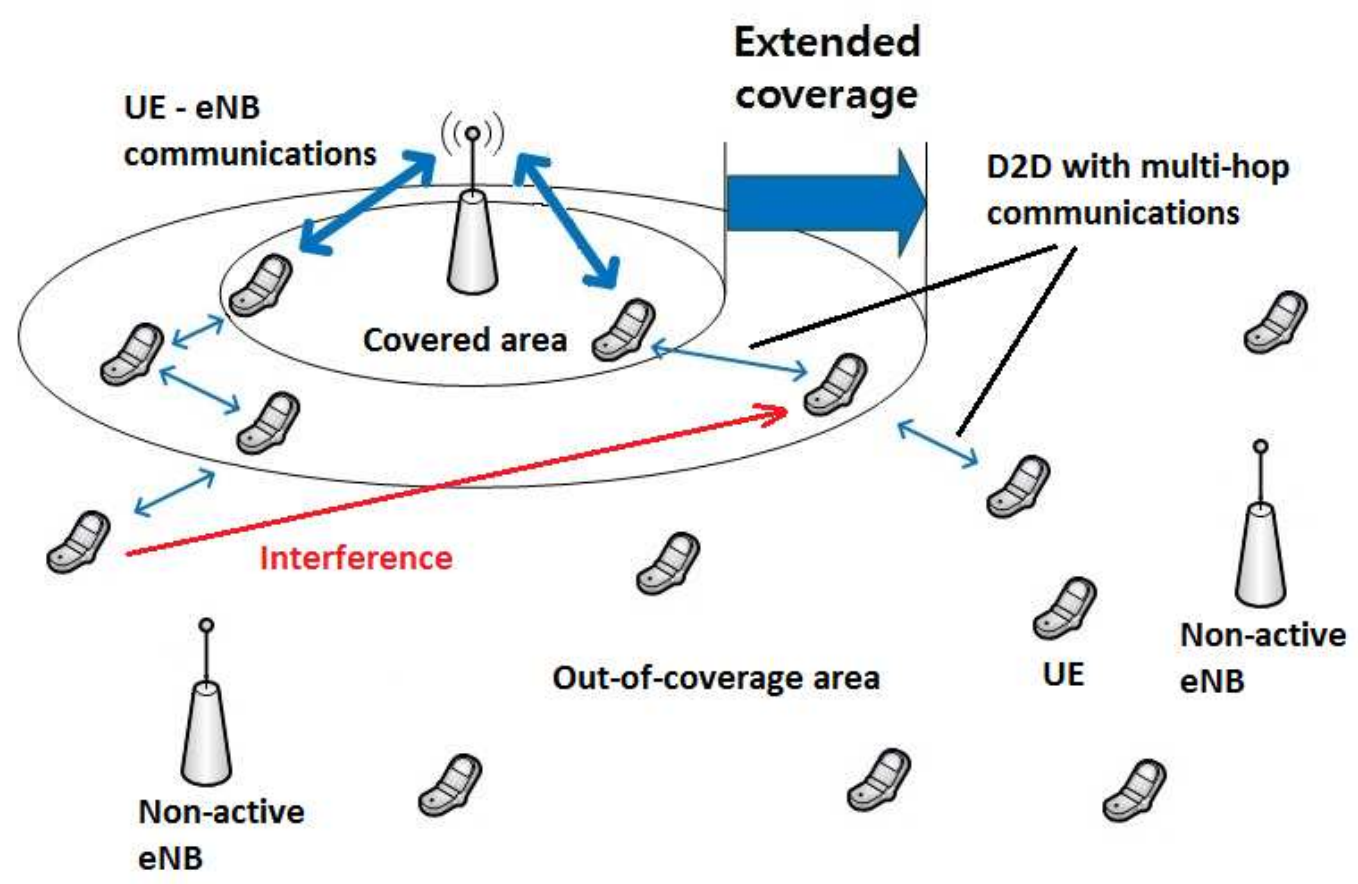

Figure 1.1: Extended coverage using D2D and multi-hop in public safety communications scenario with partial coverage.

areas [22]. ICIC and power control techniques has to be applied in order to reduce interference between D2D links and to/from cellular network in the case of underlying D2D discovery and communications. Also, EE and SE performance of D2D has to be evaluated since for emergency situations, efficient energy and spectral resources utilization are critical.

\subsection{Research Objectives}

The research plan of this project is designed based on the aforementioned challenge that represents the MBS coverage extension based on D2D communications and multihop for the case of partial coverage during emergency situations. The objectives of this research are categorized into three research trusts: 
- Literature survey on 3GPP LTE ProSe: A deep literature survey will help to describe the most important aspects of D2D discovery and communications, proximity services and multi-hop techniques.

- D2D discovery performance evaluation: Using a 3GPP compliant LTEAdvanced (LTE-A) heterogeneous network (HetNet) system level simulator we can study and describe the performance of D2D discovery process in presence on inter-cell interference after applying ICIC techniques and smart discovery algorithms. Also, the improvements of D2D discovery by using multi-hop UE cooperation has to be evaluated.

- D2D communications performance evaluation in public safety partial coverage scenarios: Using a modified version of the 3GPP D2D simulator, we can obtain simulation results about the real impact of the use of D2D communications and multi-hop techniques in extending the coverage of macro base stations during emergency situations, which represent a novel approach and have not been addressed in the literature so far. The routing path complexity, number of hops, and neighboring D2D link interference will directly impact the capacity, SE and EE performance of the D2D communications.

\subsection{Organization of the Thesis}

The evaluation of using D2D communications and multi-hop in extending the coverage area of the base stations deals with the complete understanding of D2D standardization, physical layer, discovery and communication processes. Chapter 2 provides a general overview about D2D and the most important aspects of this technology: channel model, standardization, resources management, use cases and evaluation metrics. Chapter 3 discusses the details about D2D discovery process and the impact of ICIC on the performance of D2D discovery in a HetNet environment, which is evaluated 
using a 3GPP compliant system level simulator. Also, the use of multi-hop techniques in improving discovery performance is evaluated. Then, chapter 4 focus the discussion on D2D communications and the benefits of using multi-hop in extending the network coverage in partial coverage public safety scenarios while maintaining the expected QoS. The impact of multi-hop techniques in the EE and SE performance is also studied. Finally, conclusions are offered as well as future work recommendations. 


\section{CHAPTER 2}

\section{OVERVIEW OF 3GPP D2D COMMUNICATIONS}

3GPP Release 12 introduces the support to D2D or direct-mode communications in LTE- Advanced (LTE-A) [23], enabling Peer-to-Peer (P2P) transmissions between devices in proximity [24-28]. D2D communications in cellular networks is defined as direct communications between two mobile users without directly involving the macro base station, however, in $3 \mathrm{GPP}, \mathrm{MBSs}$ will cooperate in peering the D2D pairs and assigning the communication resources [14,29].

D2D communications is generally non-transparent to the cellular network and it can occur on cellular spectrum (in-band) or unlicensed spectrum (out-band) [14]. The coexistence of D2D and cellular transmission has been mentioned in literature studies for about ten years.

Mobile users in today's cellular network use high rate services in which they could potentially be in range for direct communications. Hence, D2D communications in such scenarios can highly increase the spectral efficiency of the network, and can potentially improve the throughput, energy efficiency and delay. D2D communications is also a way to allow UEs to act as relays for each other. At any particular moment, some UEs can act as an underlay of wireless relays to each other, combining together with the MBS and forming which is known as a temporary heterogeneous network (HetNets) $[14,19]$. As mentioned before, in emergency situations, users located in non-covered areas can benefit from these HetNets since they can act as extensions of active cells.

Nokia was one of the first companies leading the efforts in investigating on D2D communications as an underlay to a cellular network. In one of their proposals, the authors reported gains from D2D communications in terms of sum rate improvements 


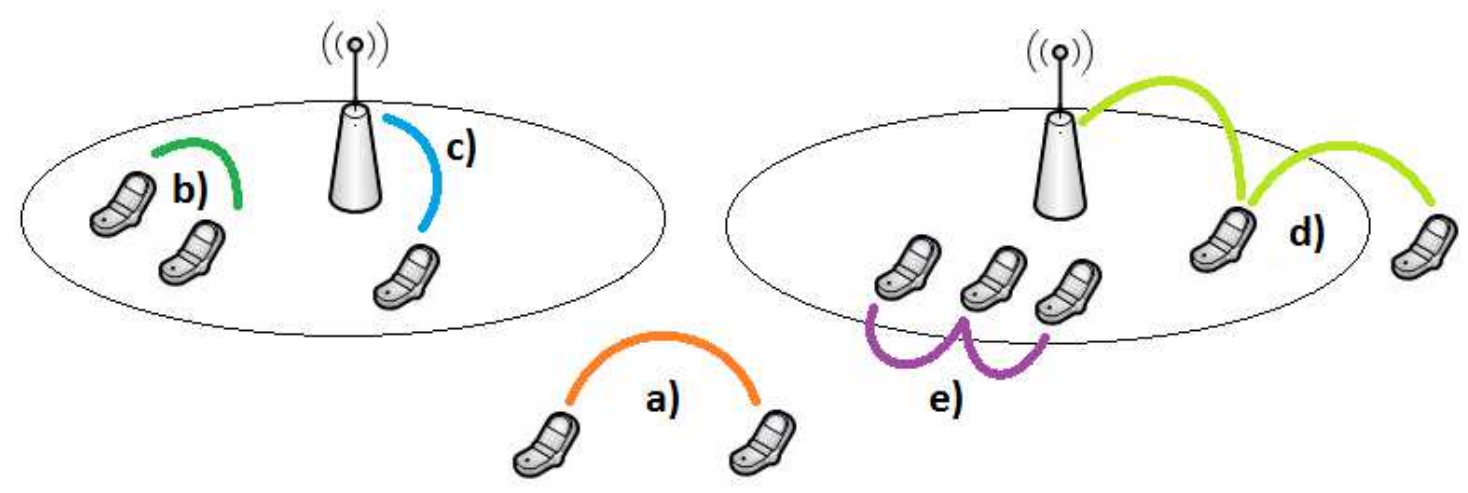

Figure 2.1: D2D communications as an underlay to a cellular network for public safety: a) locally routed D2D communications, b) D2D communication without network support, c) LTE communication path for UEs in MBS coverage, d) UE to network relay for out-of coverage UE in public safety scenario, e) D2D direct communication in public safety scenario.

in a single cell scenario. The overall throughput in the cell is maximized by choosing optimal spectrum sharing mode between D2D and cellular UEs [30].

Other studies has shown that by using power optimization methods and resource allocation is possible to mitigate interference between D2D and macro links and intracell D2D-to-downlink (DL) and uplink (UL)-to-D2D. This is one of the advantages of in-band D2D if compared with the out-band case. The D2D underlay can be optimized while a target performance level of the cellular network is maintained [31$33]$.

\subsection{Introduction to D2D Proximity Services}

With the popularity of smart devices, and the potential huge market of proximitybased services (ProSe) and applications, there is an urgent need to integrate D2D mode transmissions in the next-generation cellular network to enable efficient discovery and communication between proximate users, and to eventually provide ubiquitous connections and a rich range of services to mobile users. 
From a technical perspective, exploiting the nature of proximity may provide multiple benefits. First, D2D UEs may enjoy high data rate and low end-to-end delays. Second, if compared with normal cellular communication, direct communications saves energy and improve radio resource utilization. Third, switching from an infrastructure path to a direct path offloads cellular traffic, alleviating congestion. Finally, other benefits may directly impact public safety networks, such as cellular range extension via UE-to-UE relaying.

D2D potential applications based on mobile user proximity can be categorized as in Table $2.1[14,26]$.

\subsubsection{D2D Key Functions}

Some of the essential functions that allow LTE D2D to address the above-mentioned potential services are:

1. D2D discovery: Allows devices in physical proximity to discover each other using LTE radio technology. In the general case, this discovery process is performed within LTE network coverage and under the control of the network operator. Discovery in regions with partial and non-coverage is also desired. In this case, UEs must support larger coverage range and, since there is not network controller, it will be required to periodically transmit/receive discovery signals with basic information about identification, location, channel status etc, which directly impacts UEs' battery life. D2D discovery is of critical importance as it allows the initiation of any D2D communication and enables proximitybased services [24,34]. However, in high interference environments (e.g. HetNet deployments) UE-to-UE discovery can be affected by the influence of other stations (i.e. other UEs, macro and picocells) which utilize the same frequency bands [35]. Normally, a signal-to-noise-plus-interference-ratio (SINR) threshold 
$\gamma_{\text {thresh }}$ defines the discovery region of a UE in any proposed D2D discovery method, so, it is clear that the interference between D2D and the base stations will affect the discovery process in those cases where D2D occurs in-band $[36,37]$.

2. D2D data communication: Allows data path happening directly between proximate D2D UEs instead of passing through the base stations. However, some control from the operator could be required for data/traffic protection and secure transmissions. Also, the operator's control enables a QoS framework which provides differential communication treatment based on D2D services, channel conditions, subscribers, device technology etc.

3. D2D relay: Allows multi-hop links to be formed between two D2D devices or between the cellular infrastructure and an endpoint UE. D2D with UE relay enhances data throughput of cell-edge users and also can used to connect faraway UEs with no cellular coverage to the base stations, so it will extend the cellular coverage.

In chapter 3 and 4 , some of the above-mentioned functions will be detailed.

\subsection{GPP D2D Standardization}

The first steps on integrating D2D to LTE-Advanced (LTE-A) were made by Qualcomm. They developed a technology called FlashLinq that allows cellular devices to automatically and continuously discover thousands of other FlashLinq enabled devices within $1 \mathrm{Km}$ and communicate, peer-to-peer, without the need of intermediary infrastructure [20]. FlashLing is a synchronous Time Division Duplex Orthogonal Frequency-Domain Multiple Access (TDD OFDMA) technology operated in dedicated license spectrum and is featured by its high discovery range, discover capacity and distributed interference management. Qualcomm planned to adapt FlashLinq to 


\begin{tabular}{|c|c|}
\hline Category & Application \\
\hline Commercial/Social & $\begin{array}{l}\text { - Interactive local guidance: Interactive guidance for } \\
\text { customers, tourists, commuters and other users of com- } \\
\text { mercial services. For example, advertisements from } \\
\text { nearby stores and restaurants, flight and subway infor- } \\
\text { mation, vacancy in parking lots, etc. } \\
\text { - Connection to Machine-to-Machine (M2M)/ } \\
\text { Vehicular-to-Vehicular (V2V): D2D-enabled de- } \\
\text { vices can serve as controllers and or gateways of M2M } \\
\text { and V2V networks. } \\
\text { - Social discovery: Discovery of nearby persons linked } \\
\text { by social networks with mutual interests. } \\
\text { - Entertainments: Involves a large variety of personal } \\
\text { devices, such as mobile smart devices, game consoles, } \\
\text { cameras, Tvs etc. Typically for content sharing, local } \\
\text { gaming or multicasting. }\end{array}$ \\
\hline Enhanced networking & $\begin{array}{l}\text { - Traffic offload: From cellular infrastructure network } \\
\text { to D2D link when the two endpoints devices are in prox- } \\
\text { imity. The D2D offloading might alleviate network con- } \\
\text { gestion, enforce the link quality and reduce the power } \\
\text { use between two proximate devices. } \\
\text { - Coverage extension: One or more devices can obtain } \\
\text { access to an infrastructure network (internet or cellu- } \\
\text { lar network) through assistance of one or more devices } \\
\text { that act as relays or access gateways. This can provide } \\
\text { network coverage to those devices that have poor or no } \\
\text { network connectivity - such as in indoor environments, } \\
\text { at the edge of MBS, or in case of partial coverage due } \\
\text { an emergency situation in public safety scenarios. }\end{array}$ \\
\hline
\end{tabular}

Table 2.1: Potential applications of D2D based on mobile user proximity.

the $3 \mathrm{GPP}$ architecture using the LTE radio interface and proposed D2D in LTE-A as a study item. To this purpose the work item called ProSe in 3GPP TSG-SA1 was completed in May 2013 [38].

In 2014, TSG SA2 (Architecture working group) was also completed. This group was created to evaluate possible $3 \mathrm{GPP}$ technical solutions for architectural enhance- 
ments needed to support ProSe based on the SA1 service requirements. Radio Access working group (TGS RAN1) has been also completed in 2014 defining the necessary support in the LTE radio interface. This working group included two subfeatures: ProSe discovery and ProSe communications.

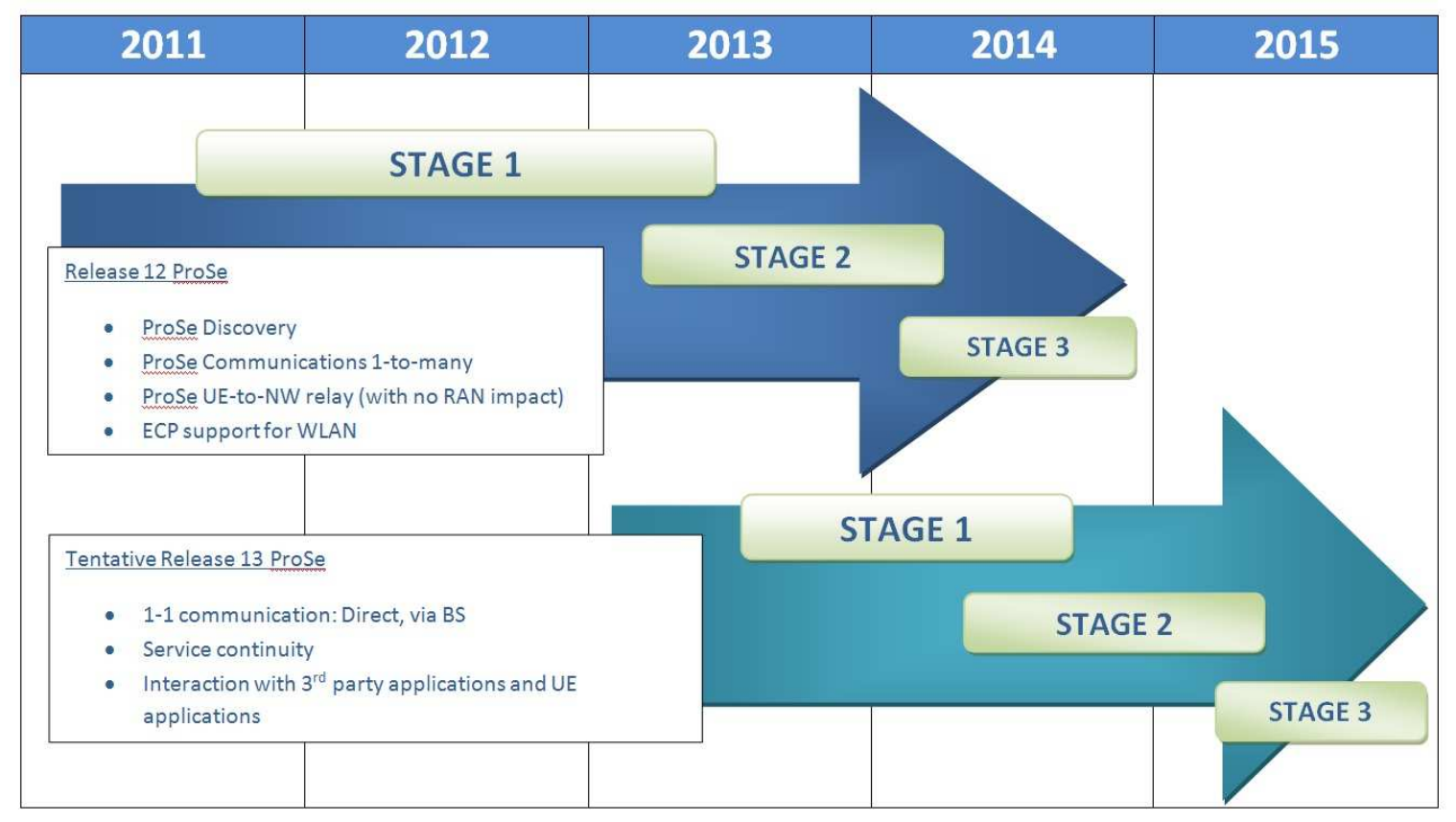

Figure 2.2: D2D standarization roadmap [2].

Feasibility study for proximity services was presented in TR 22.803 [1] with the purpose of identify use cases and potential requirements for discovery and communications between UEs that are in proximity, including network operator control, authentication, authorization, accounting and regulatory aspects. This document serves as an essential guidance for D2D system design. The study summarize use cases and scenarios concerning general commercial/social use, network offloading as well as public safety.

Table 2.2 summarizes general and public safety use cases [2]. 


\begin{tabular}{|c|c|}
\hline Use case type & Content \\
\hline Commercial/Social & $\begin{array}{l}\text { - Restricted ProSe discovery } \\
\text { - Open ProSe discovery } \\
\text { - Discovery use case with subscribers from different } \\
\text { PLMNs } \\
\text { - Discovery use case with roaming subscribers } \\
\text { - Network ProSe discovery use case } \\
\text { - Service continuity between infrastructure and E-UTRA } \\
\text { ProSe communication paths } \\
\text { - Operator A uses ProSe to enhance location and presence } \\
\text { services } \\
\text { - ProSe for large numbers of UEs } \\
\text { - ProSe-assisted WLAN direct communications use case } \\
\text { - Service management and continuity for ProSe-assisted } \\
\text { WLAN direct communications } \\
\text { - Use case for ProSe application provided by the third- } \\
\text { party application developer } \\
\text { - Concurrent E-UTRAN infrastructure and WLAN prox- } \\
\text { imity communication } \\
\text { - Network offloading via WLAN ProSe communication }\end{array}$ \\
\hline Public Safety & $\begin{array}{l}\text { - ProSe discovery within network coverage. } \\
\text { - ProSe discovery out of network coverage. } \\
\text { - Can discover but not discoverable. } \\
\text { - Basic ProSe one-to-one direct user traffic initiation in } \\
\text { public safety spectrum. } \\
\text { - UE with multiple one-to-one direct user traffic sessions } \\
\text { in public safety spectrum. } \\
\text { - ProSe group. } \\
\text { - ProSe broadcast. } \\
\text { - ProSe relay. } \\
\text { - ProSe hybrid and range extension. } \\
\text { - ProSe range. } \\
\text { - Public safety implicit discovery. } \\
\text { - Co-existence of ProSe communication and E-UTRAN } \\
\text { communication. }\end{array}$ \\
\hline
\end{tabular}

Table 2.2: ProSe use cases and scenarios defined in TR 22.803 [1]. 


\subsection{Long Term Evolution D2D Communications Resource Use}

The transmission resource in LTE possess dimensions of time, frequency and space. The spatial dimension is accessed by means of multiple antennas ports at the MBS; for each antenna porta Reference Signal (RS) is provided to enable the UE to estimate the radio channel.

The time-frequency resources for each transmit antenna port are subdivided according to the following structure: the largest unit of time is the 10 ms radio frame, which is subdivided into ten $1 \mathrm{~ms}$ subframes, each of which is split into two $0.5 \mathrm{~ms}$ slots. Each slot comprises seven Orthogonal Frequency Division Multiplex (OFDM) symbols in the case of normal Cyclic Prefix (CP) length, or six if extended CP is used. In the frequency domain, resources are grouped in units of 12 subcarriers, occupying a total of $180 \mathrm{kHz}$ with a subcarrier spacing of $15 \mathrm{kHz}$. The unit of 12 subcarriers for a duration of one slot is termed Resource Block (RB). The smallest unit of resource is the Resource Element (RE), which consist of one subcarrier for a duration of one OFDM symbol [39].

This resource structure enables a scheduler to assign resources dynamically and flexibly, based on time-variant frequency selective channel for each user, and thus makes it possible to achieve high spectral efficiency and QoS of each individual.

In principle, D2D may use a combination of UL and DL spectrum for Frequency Division Duplex (FDD) and UL and DL subframes for Time Division Duplex (TDD).

In TDD, the traditional half-duplex UE antenna put constraints in D2D transmissions. UEs in D2D mode, cannot receive downlink signals from the base station at the same time while, in uplink, D2D devices cannot transmit signals to the base station. A costly solution could integrate full duplex antenna to UEs, but besides the cost, 
leakage and antenna size represent obstacles that cannot be ignored. An alternative solution can be time resource partition for cellular and D2D transmissions, but at the expense of spectral efficiency gain.

In FDD, for UEs transmitting in the same band as cellular result quite challenging since interference can be severe when a UE receives (or transmit) form (to) the base station on the DL (UL) band, while is transmitting (receiving) to (from) another D2D UE in the same band. For in-band transmissions, it is possible to allocate dedicated resources (degrading spectral efficiency gain), or to allow overlapping resources between D2D and cellular transmissions in which case interference coordination is critical.

\subsection{GPP D2D Evaluation and Channel Models}

For standardization purposes, even when several uses and scenarios have been defined for D2D and ProSe applications, a generic design of D2D discovery and transmission procedures is preferred. In this context, three coverage scenarios can be identified (for the simplest case of one-hop D2D communication):

- In coverage: Both D2D devices are under network coverage.

- Out of coverage: Both D2D devices are out of network coverage.

- Partial coverage: One of the two D2D devices is out of network coverage. This design case is common during emergency situations, when some of the base stations are shut down due the impact of the disaster or because of power outage.

For the case of in coverage D2D, it is required that discover and communication process occur under the control of the network operator, this way, the interference caused by $\mathrm{D} 2 \mathrm{D}$ over regular cellular transmission will be manageable. In other words, the 
network would be able to identify, authenticate and authorize D2D UEs participating in discovery, and to determine resources and power of D2D transmissions. A variation of the previous case scenario is when D2D transmitter and receiver are located in different operator networks. Inter-operator scenario and out-of-coverage scenario implies that D2D devices might not be originally synchronized to each other, and the initialization of D2D link should be able to act in asynchronous mode.

Another important aspect when analyzing D2D design principles is that, in order to minimize integration impacts, D2D would have to reuse as much as possible the current LTE PHY and MAC features.

3GPP D2D studies involve complex design/research topics and require comparison of different technical options. In [40], modeling assumptions (including system bandwidth, UE density and mobility, traffic models and RF parameters) as well as performance metrics are defined. 3GPP continues the debate about network layout for evaluating D2D. There are six (6) different evaluation options [34] already defined:

- Option 1: Urban macro (500 m inter-site distance (ISD)) + 1 remote radio head (RRH)/Indoor Hotzone per cell.

- Option 2: Urban macro (500 m ISD) + 1 Dual stripe building per cell.

- Option 3: Urban macro (500 m ISD) with all UEs outdoor.

- Option 4: Urban macro (500 m ISD) + 3 RRH/Indoor Hotzone per cell.

- Option 5: Urban macro (1732 m ISD).

- Option 6: Urban micro (100 m ISD).

All layout options consider a hexagonal grid, 3 sectors per site with 19 or 7 macrocell sites. The total number of active UEs per cell are specified as 25 for options 1, 2, 4 and 10 for options 3,5 and 6 . 


\subsubsection{GPP UE Dropping in D2D Scenarios}

UE dropping for public safety and non-public safety scenarios are different. In public safety, layout option 5 form the previous list is selected as mandatory, while option 3 and 1 are specified as optional layouts. For other general scenarios layout option 1 is selected as mandatory, while other options except 5 are selected as optional.

For simulation layout options 1,2 and 4, two-third of the UEs are uniformly randomly dropped whiting the clusters of small cells, while one-third of the UEs are uniformly randomly dropped throughout the macro geographical area, with $20 \%$ of the UEs being outdoors and $80 \%$ of the UEs being indoors. On the other hand, for layout options 3,5 and 6 the UEs are dropped in two different ways:

1. Uniform drop: All UEs are dropped uniformly throughout the MBS geographical area.

2. Hotspot drop: Within each macrocell area, a hotspot circular region is defined with radius of $40 \mathrm{~m}$ and randomly distributed location. two-third of the UEs are uniformly randomly distributed within this hotspot are and the rest one-third is uniformly randomly distributed outside the hotspot area.

\subsubsection{GPP Device-to-Device Evaluation Metrics}

Different metrics are agreed upon to evaluate the performance of D2D discovery and communication techniques. For discovery purposes, the following metrics are defined, and can be used for in-network, partial network, out-of-network and non-public safety cases.

- Performance target: For open discovery represents the number of UEs discovered as a function of time and the Cumulative Distribution Function (CDF) of num- 
ber of discovered UEs. For closed discovery, where the UE to be discovered is known, the discovery probability as a function of time is defined.

- Range and reliability: Probability of discovery vs pathloss and the probability of false alarm.

- Impact on Wide Area Network (WAN): Amount of resources used.

- Power consumption: Modeled through on-time or equivalent power consumed.

For D2D communications the following evaluation metrics are defined:

- D2D throughput and spectral efficiency: User throughput and perceived user throughput for full buffer and VoIP system capacity.

- Range and reliability: Performance vs pathloss or D2D link distance.

- Impact on WAN Change in cell throughput/cell spectral efficiency and CDFs of perceived per-user throughput with and without D2D.

- Power consumption: For both in-coverage and out-of-coverage scenarios.

\subsubsection{GPP D2D Channel Propagation Model}

Appropriate channel models are important for generating realistic results for D2D evaluation in both, link and system simulations. Specifically, the following factors make the propagation characteristics of UE-UE D2D links distinct from BS-UE links $[26]$.

- Dual mobility: In BS-UE links, only the UEs are mobile while the base stations are fixed. In contrast, both terminals may be mobile in UE-UE links, creating a dual mobile scenario. This dual mobility affects the temporal correlation of shadowing as well as fast fading, increasing Doppler spread. 


\begin{tabular}{|c|c|c|c|}
\hline & $\begin{array}{l}\text { Outdoor to } \\
\text { outdoor }\end{array}$ & Outdoor to indoor & Indoor to indoor \\
\hline Pathloss & $\begin{array}{c}\text { ITU-1411-6 or } \\
\text { Winner+ B1 }\end{array}$ & $\begin{array}{c}\text { Dual strip, or } \\
\text { Winner+ B4, or } \\
\text { Winner II A2 }\end{array}$ & $\begin{array}{c}\text { Dual strip, or InH } \\
\text { (TR 36.814, or } \\
\text { Winner II A1) }\end{array}$ \\
\hline $\begin{array}{c}\text { LOS } \\
\text { probability }\end{array}$ & ITU-R IMT UMi & ITU-R IMT UMi & $\begin{array}{l}\text { ITU-R IMT UMi, } \\
\text { or ITU-R IMT } \\
\text { InH, or Winner II } \\
\text { A1 }\end{array}$ \\
\hline Shadowing & $\begin{array}{c}7 \mathrm{~dB} \text { log-normal, } \\
\text { or } 10 \mathrm{~dB} \\
\log \text {-normal }\end{array}$ & $7 \mathrm{~dB}$ log-normal & $\begin{array}{c}\text { LOS: } 3 \mathrm{~dB} \\
\text { log-normal, or } \\
\text { NLOS: } 4 \mathrm{~dB} \\
\text { log-normal }\end{array}$ \\
\hline Fast fading & $\begin{array}{l}\text { ITU-R IMT UMi } \\
\text { LOS and NLOS }\end{array}$ & $\begin{array}{c}\text { ITU-R IMT UMi } \\
\text { O2I }\end{array}$ & $\begin{array}{l}\text { ITU-R IMT InH } \\
\text { LOS and NLOS }\end{array}$ \\
\hline
\end{tabular}

Table 2.3: Channel model assumptions for simulating D2D scenarios.

- Low antenna height: The antenna height at the BS may range from several meters to tens of meters, while the typical antenna height at the UE is $1.5 \mathrm{~m}$. With the same link length, UE-UE links incurs higher pathloss than BS-UE links. Also, scattering environment in D2D is different if compared with the typical BS-UE case.

- Interlink correlation: It is expected that D2D UEs are of high density. As a result, small inter-UE distnaces are expected; and compared to BS-UE links, there will be much higher correlations in the propagation characteristics of UEUE links including shadowing, angle of arrival (AoA) and angle of departure (AoD) spreads and delay spreads.

The main difference between the UE2UE channel and the BS2UE channel lies in the dual mobility of both devices in the former case [34,35], according to which the Doppler frequency calculation changes.

Based on the aforementioned characteristics of D2D links, 3GPP agreed to adapt existing channel models to D2D, summarized in Table 2.3. 


\section{CHAPTER 3}

\section{D2D DISCOVERY AND INTER-CELL INTERFERENCE COORDINATION IN LTE-A HETEROGENEOUS NETWORKS}

UE discovery is a procedure where UEs find each other periodically before the communication link is set up. For that purpose, UEs have to exchange predefined signals, referred to as beacons. By checking beacons periodically, a UE maintains a list of other UEs in proximity, in order to establish communication link when it is needed [41].

In [34], the authors presented a D2D system level simulation environment based on the 3GPP simulation assumptions and a performance evaluation of three different D2D discovery algorithms.

In this chapter, we extend the aforementioned D2D system level simulator to a heterogeneous cellular network environment based on 3GPP simulation assumptions for HetNets [42]. The considered cellular network is depicted in Fig. 3.1, and consists of macrocells, picocells, regular UEs (communicating with macrocells/picocells), and D2D UEs. In this new model, we included macrocell and picocell-to-UE interference

analysis in addition to the new ICIC approach and the use of multi-hop. Even though D2D discovery is considered for the uplink transmission mode in 3GPP, we focus on the downlink transmission due to the possibility of re-using the existing enhanced intercell interference coordination (eICIC) techniques recently developed for HetNets $[42,43]$.

\subsection{Introduction to D2D Discovery Process}

To fulfill the requirements for the purpose of D2D discovery over the air, two functions are essentials: 


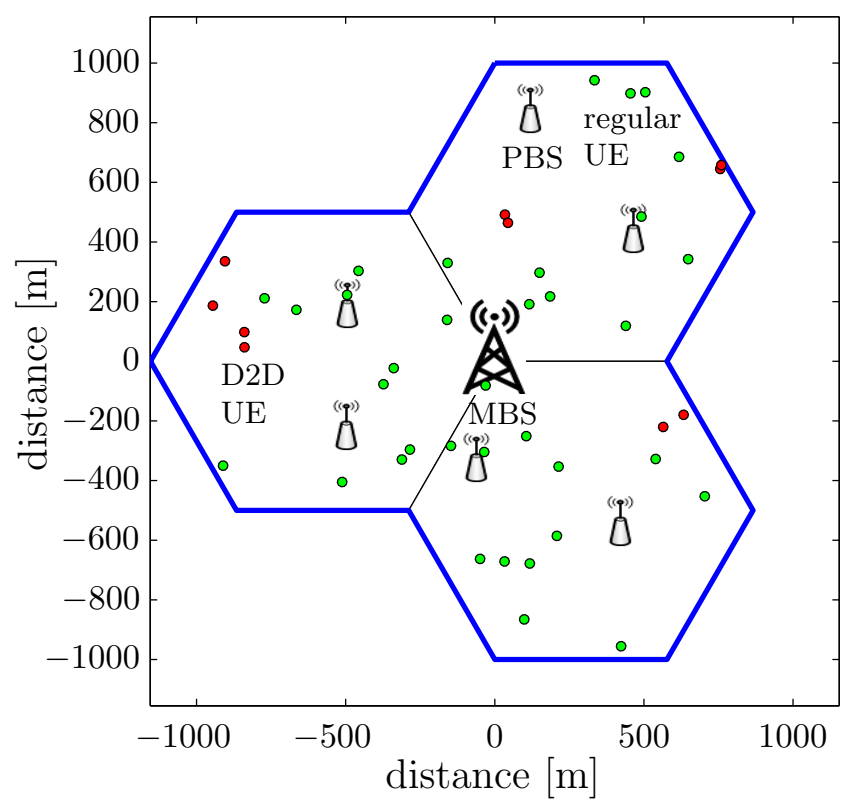

Figure 3.1: Layout option 5 according to R1-131755 overlaid by small cell BSs.

- Proximity detection: UEs are aware of other UEs in proximity supporting D2D.

- Identity detection: UEs can identify other UEs in proximity supporting D2D.

D2D discovery starts with proximity detection, followed by identity detection. To fulfill the first step, a scan/search mechanism using beacon sequences is implemented. Since UEs can be located in different network operators or in out-of-coverage areas, UEs must to be able to decode these beacon sequences asynchronously. It is preferred that synchronization can be achieved through beacon sequences at the initial stage of D2D discovery so that subsequent messages can benefit from synchronized transmissions [38].

After the transmission of beacon sequence for proximity detection, there are two way to convey UE identity associated with the beacon sequence: by transmitting a subsequent message containing its identity or via network signaling. The first case offers a discovery solution with simpler signaling. The second case however, introduces additional overhead since the network has to receive first the UE identity through the 
initial beacon and then, report back the UE identity to other UEs using higher level signaling. In case the discovering or discovered UE are out-of-network, the identity detection will need special mechanisms like using another UE as relay to gain access to the network or by using an alternative UE-to-UE identity exchange mode, exclusively designed for out-of-coverage UEs.

Due to network dynamics such as UE mobility and UE On/Off status, the number and composition of UEs in proximity will change over time. In order to maintain updated the list of UEs in proximity, the discovery procedure has to be performed regularly in time. Also, for allowing an efficient discovery, a UE which wants to be discoverable should be able to perform discovery whether it is in RRC_CONNECTED or $R R C \_I D L E$ state.

Another important aspect to keep in mind while performing discovery is that, if UEs are in half duplex mode, the UE transmitting discovery signals cannot receive beacons transmitted by other UEs. This leads to the need of arranging transmissions and receiving scheduling so the UE transmitting can detect other UEs doing discovery in a reasonable time. In [38], the author describes an power efficient scheduling

method, where half of the UEs in a group are transmitting while the other half is listening. In the next time instant the roles can be interchanged, so two UEs will not always transmit at the same time and fail to detect each other.

The resource use of D2D discovery signal is key design factor. There are several choices that has to be analyzed [38]:

\section{D2D uses dedicated or overlapping resources with cellular transmis-} sion: For dedicated resource utilization interference from D2D can be avoided, whereas if D2D uses overlapping resources with cellular network, interference management can be challenging. 
2. D2D performs discovery in UL or DL: D2D can operate in both UL and DL resources. however, using UL brings several advantages. First, implementation cost is reduced because of the reuse of the existing LTE UE RF transceiver; second, regulations constraints existing in some countries which obstruct UEs in FDD deployment from transmitting in DL are avoided.

3. Multiplex between D2D discovery signal and cellular in case of dedicated resources: When operating in UL resources, D2D and UL cellular transmissions can either be frequency or time multiplexed. First case implies that discovery resources are spread out in time domain which leads to inefficient power consumption since UEs has to be awake for longer periods of time.

4. Multiplex between several D2D discovery signals: A UE participating in discovery is often interested in discovering all the UEs in proximity. The signal received from multiple UEs can be time multiplexed (resource inefficient), frequency multiplexed (suitable for large-scale discovery activity) or code multiplexed (vulnerable to near-far problem).

\subsection{D2D System Level Simulation Environment}

The D2D system level simulator including a heterogeneous cellular network deployment is based on the assumptions in 3GPP LTE-Advanced, including those on the radio frame structure, the physical resource structure and the channel modeling $[42,44,45]$.

We developed a D2D system level simulator consisting of modular simulation blocks as shown in the flowchart in Fig. 3.2. For adapting the D2D simulator to the assumptions in 3GPP LTE-Advanced, we modified the UE-to-UE initial channel propagation model and introduced the Macro-to-UE and Pico-to-UE multi-path links. These modifications directly impacted the Initialization, Channel generation 
and SINR calculation blocks. For path loss, shadowing and fast fading calculation in the new scenarios we considered the channel models provided in [42].

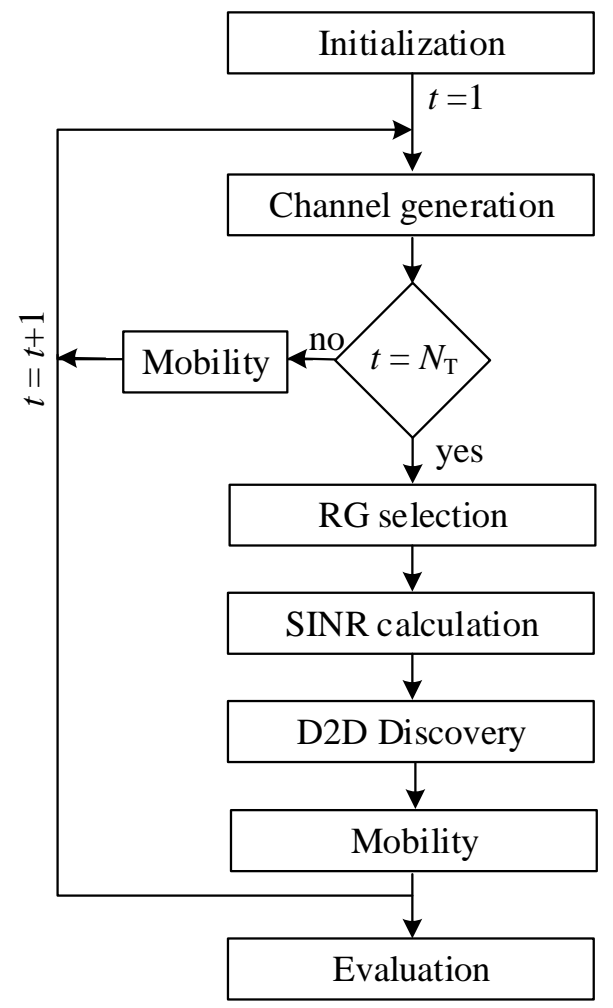

Figure 3.2: D2D system level simulator flowchart.

The D2D system level simulator continuously simulates the temporal development of a LTE-Advanced based cellular network by calculating densely spaced snapshots, each corresponding to one subframe $t$ with an iteration step size of $T=1 \mathrm{~ms}$. Within each snapshot, the position of each UE changes according to its velocity, and the channels between each macro/pico base station (BS) to each UE and from each UE to each UE are newly calculated. D2D discovery is performed in each discovery time slot over $N_{\mathrm{F}} \times N_{\mathrm{T}}$ discovery resources (DR) based on different discovery methods,

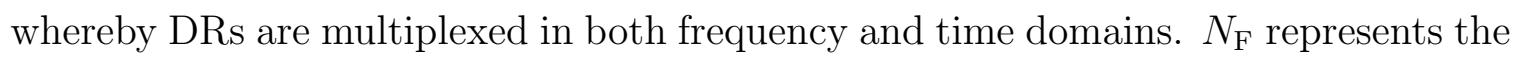
number of available DRs in the frequency domain and $N_{\mathrm{T}}$ is the number of discovery 
subframes in the time domain. The main blocks of the system level simulator will be described in the following subsections.

\subsubsection{Initialization}

In this simulation block the simulation parameters, discovery methods, and ICIC algorithms are set. The simulation parameters include the number of macrocells, the system bandwidth according to 3 GPP LTE specifications, the carrier frequency $f_{0}$, the transmit powers, the duplex mode (full duplex or half duplex), the number of discovery subframes $N_{\mathrm{T}}$. Also, the layout option for D2D discovery and communication based on [44] and, on the selected layout option, the number of UEs, and the UE distribution (uniform/hotspot).

\subsubsection{Channel Modeling}

Time and frequency selective channel models considering multi-path fading effects are implemented. The simulator supports two different channel modeling methods for the BS to UE (BS2UE) channels and for the UE2UE channels according to [44]. All path loss models, shadowing factors/correlation, antenna gain models and multipath parameters are based on 3GPP assumptions. We consider that the total bandwidth $(\mathrm{BW})$ is divided into subchannels with bandwidth $\Delta f=15 \mathrm{kHz}$. Orthogonal frequency division multiplexing (OFDM) symbols are grouped into resource blocks (RBs). Macro and picocells operate in the same frequency band and have the same amount $N_{\mathrm{RB}}$ of available RBs, while UEs perform D2D discovery in the same frequency band, too. The main difference between the UE2UE channel and the BS2UE channel lies in the dual mobility of both devices in the former case, according to which the Doppler frequency calculation changes. For a multi-path channel with $N$ paths 
and $L$ subpaths for each path, the Doppler frequency for the $l \in L$-th subpath of the $n \in N$-th path of the BS2UE channel is given by $[44,46]$ :

$$
f_{\text {Doppler }, n, l}^{\mathrm{BS} 2 \mathrm{UE}}=\left[\left\|v_{\mathrm{rx}}\right\| \cos \left(\phi_{n, l}^{\mathrm{AoA}}-\theta_{\mathrm{vrx}}\right)\right] \frac{f_{0}}{c},
$$

with $\left\|v_{\mathrm{rx}}\right\|$ the velocity of the receiver/UE, $\phi_{n, l}^{\text {AoA }}$ the angle of arrival from the $l$-th subpath of the $n$-th path, $\theta_{\mathrm{vrx}}$ the random angle receiver's/UE's direction of movement, and $c=3 \cdot 10^{8} \mathrm{~m} / \mathrm{s}$.

The Doppler frequency for the $l \in L$-th subpath of the $n \in N$-th path of the UE2UE channel is defined as [44]:

$$
f_{\text {Doppler }, n, l}^{\mathrm{UE} 2 \mathrm{UE}}=\left[\left\|v_{\mathrm{rx}}\right\| \cos \left(\phi_{n, l}^{\mathrm{AoA}}-\theta_{\mathrm{vrx}}\right)+\left\|v_{\mathrm{tx}}\right\| \cos \left(\phi_{n, l}^{\mathrm{AoD}}-\theta_{\mathrm{vtx}}\right)\right] \frac{f_{0}}{c},
$$

with $\left\|v_{\mathrm{tx}}\right\|$ the velocity of the transmitter/UE, $\phi_{n, l}^{\mathrm{AoD}}$ the angle of departure from the $l$-th subpath of the $n$-th path, and $\theta_{\mathrm{vtx}}$ the random angle of direction of the transmitter/UE. The output of this simulation block is the channel transfer function from each transmitter to each receiver over all RBs and is calculated in each subframe $t$.

\subsubsection{Resource Group Selection}

Different resource group selection algorithms are supported by the D2D system level simulator. The RG selection algorithms are described in Section 3.4. 


\subsubsection{Signal-to-Interference-plus-Noise-ratio Calculation}

After each UE selects its RG based on a preselected algorithm, the SINR is calculated for each UE on its selected RG. Hereby, the total interference is considered, i.e. the interference from macro/pico BSs and UEs performing discovery on the same RG.

\subsubsection{D2D Discovery algorithms}

After each UE selects its resource group (RG) based on a preselected algorithm, the D2D discovery is performed based on a variable SINR threshold value, i. e. a UE is discovered when its SINR is larger than a SINR threshold. This simulation block can be extended to various D2D discovery approaches.

\subsubsection{Mobility}

A random walk process is considered, whereby all UEs in the network are assumed to be mobile. The velocity $v_{u}$ of a particular UE $u$ can be set to correspond to any of $\{3,30,60,120\} \mathrm{km} / \mathrm{h}$ as proposed in [47] or is set according to 3GPP D2D layout option assumptions. The location $\mathbf{l}_{u}(x, y, t)$ of $\mathrm{UE} u$ in position $(x, y)$ at a velocity $v_{u}$ and a random direction of movement $\mathbf{d}_{u}=[\cos (\phi), \sin (\phi)]$ with $\phi \in[0 ; 2 \pi]$ is updated in snapshot $t$ according to:

$$
\mathbf{l}_{u}(x, y, t T+T)=\mathbf{l}_{u}(x, y, t T)+v_{u} \cdot T \cdot \mathbf{d}_{u},
$$

\subsubsection{Evaluation}

The following metrics that are agreed upon to evaluate the performance of D2D discovery are considered [40]: 
- Open discovery: Total number of UEs discovered as a function of time and the cumulative distribution function $(\mathrm{CDF})$ of total number of UEs discovered as a function of time.

- Closed discovery (i.e. knowing the UEs to be discovered): Discovery probability as a function of time.

- Average number of discovered UEs vs different number of DRs.

The evaluation block is the last simulator block which is run after all snapshots. It provides averaged results over various random drops.

\subsection{D2D Interference Coordination}

In overlapping resource use, interference avoidance is challenging. It is possible to avoid intra-cell interference by assigning orthogonal resources between cellular and D2D transmissions in the same cell. On the other hand, inter-cell interference (ICI) between cellular and D2D in neighbor cells, requires cooperation between base stations. Precisely, the performance gain of the overlapping resources use scheme, depends on the interference management technique applied.

In homogeneous networks, the main mechanisms for ICIC is normally assumed to be frequency-domain based. Base stations can interchange messages over the $\mathrm{X} 2$ interface to plan about transmission on different frequency bands. Also, restrictions can be imposed on the use of resources in order to mitigate interference.

ICI becomes more critical in heterogeneous environments. LTE Rel. 10 introduced time-domain enhanced ICIC techniques, mainly focused in co-channel interference mitigation. These coordination techniques introduced the concept of Almost Blank Subframes (ABSs) in order to reduce downlink transmission power and/or cellular activity in certain subframes so the interference from MBSs towards the UEs served 
by smaller cells (picocells, femptocells) is reduced. As the picocells has much lower transmission power if compared with macrocells, the interference received from MBSs is significantly higher. The same concept can be applied to D2D for coordinating the interference received from macro and picocells in heterogeneous environments. By muting cell activity in some subframes, D2D UEs have better opportunity to use available resources without degrading the performance due high interference.

\subsection{D2D Discovery Algorithms}

It is assumed that the considered HetNet consists of a set of $\mathcal{M}=\{1, \ldots, M\}$ macrocells, a set of $\mathcal{K}=\{1, \ldots, K\}$ uniformly positioned picocells per macro sector, and a set of UEs $\mathcal{U}=\{1, \ldots, U\}$. In the considered system, the macro BS $m$ transmits with a maximum transmit power $p_{\mathrm{tx}}^{m}$, the pico BS $k$ transmits with a maximum transmit power $p_{\mathrm{tx}}^{k}$ and the UE $u$ transmits with a maximum transmit power $p_{\mathrm{tx}}^{u}$. Assuming a uniform power distribution the corresponding transmit powers per RB $r$ are $p_{\mathrm{tx}}^{m}(r)$, $p_{\mathrm{tx}}^{k}(r)$, and $p_{\mathrm{tx}}^{u}(r)$, respectively. Within the considered co-channel deployment scenario, in which the UEs perform D2D discovery in the same frequency bands as BSs are transmitting, a UE $u$ 's total received power in RB $r$ is given by:

$$
p_{\mathrm{rx}}^{u}(r)=\sum_{m=1}^{M} p_{\mathrm{tx}}^{m}(r)+\sum_{k=1}^{K} p_{\mathrm{tx}}^{k}(r)+\sum_{w=1, w \neq u}^{U} p_{\mathrm{tx}}^{w}(r) .
$$

It is also assumed that all UEs select a DR to periodically transmit their peer discovery information, whereby each DR is identified by a PDRID (Peer Discovery Resource ID). In each discovery slot with $D=N_{\mathrm{F}} \times N_{\mathrm{T}}$ DRs, each UE $u \in \mathcal{U}$ transmits its peer discovery information on one DR $d_{u} \in D$ and listens to the remaining DRs to discover its peers. Hereby, the whole BW is used for discovery, so that $N_{\mathrm{F}} \approx N_{\mathrm{RB}}$. 
a)

$R_{\mathrm{ABS}}=1$

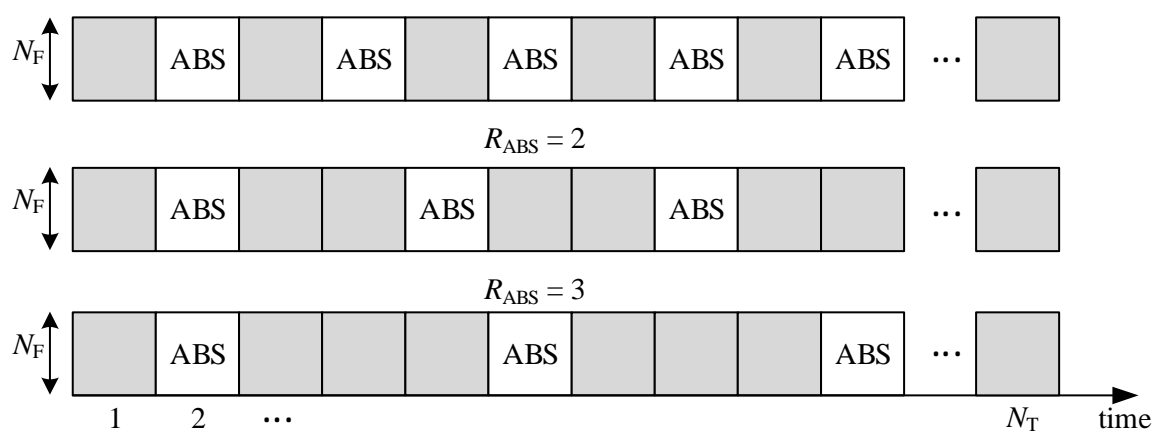

b)

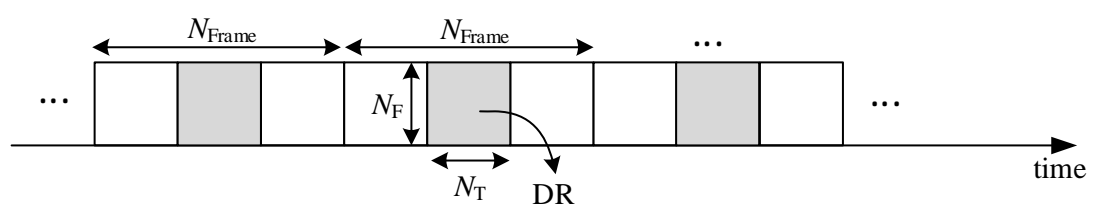

Figure 3.3: (a) Frame structure for different $\mathrm{ABS}$ rates $\left(R_{\mathrm{ABS}}\right)$, (b) DR distribution over $N_{\mathrm{T}} \times N_{\mathrm{F}}$ LTE-A subframe.

The rationale behind this is to examine a worst case scenario with full interference over the whole BW. The following two PDRID selection approaches are proposed:

- Random PDRID Selection: This is a baseline approach in which each UE $u$ selects its PDRID $d_{u}$ randomly out of $D$ DRs in each discovery slot.

- Smart PDRID Selection: In this approach, a UE $u$ considers its received powers $\mathbf{p}_{\mathrm{rx}}^{u}=\left[p_{\mathrm{rx}}^{u}(1), \ldots, p_{\mathrm{rx}}^{u}(R), \ldots, p_{\mathrm{rx}}^{u}(D)\right]$ in one discovery slot and selects its DR $d \in D$ based on the minimum received power according to:

$$
\arg \min _{d}=\left(\mathbf{p}_{\mathrm{rx}}^{u}\right) .
$$

Considering the half duplex mode, in which UEs that selected DRs within the same subframe cannot discover each other, discovery is performed based on a threshold $\gamma_{\text {thresh }}$. A UE $u$ is assumed to be successfully discovered if its SINR $\gamma^{u}(d)$ exceeds this threshold: 


$$
\gamma^{u}(d)=\frac{p_{\mathrm{tx}}^{u}(d) \cdot\left|h_{u(d), w(d)}(t, d)\right|^{2}}{\sum_{v \neq u} p_{\mathrm{tx}}^{v}(d)\left|h_{u(d), v(d)}(t, d)\right|^{2}+\mathrm{I}_{\mathrm{mac}}+\mathrm{I}_{\mathrm{pic}}+\sigma^{2}}>\gamma_{\mathrm{thresh}}
$$

with the macrocell interference

$$
\mathrm{I}_{\mathrm{mac}}=\sum_{m} p_{\mathrm{tx}}^{m}(d)\left|h_{u(d), m(d)}(t, d)\right|^{2}
$$

and the picocell interference

$$
\mathrm{I}_{\mathrm{pic}}=\sum_{p} p_{\mathrm{tx}}^{p}(d)\left|h_{u(d), p(d)}(t, d)\right|^{2}
$$

where $\left|h_{u(d), w(d)}(t, d)\right|^{2}$ is the channel gain between UE $u$ and UE $w \in \mathcal{U}$ in DR $d$ at time $t,\left|h_{u(d), m(d)}(t, d)\right|^{2}$ is the channel gain between UE $u$ and macro BS $m$ in DR $d$, and $\left|h_{u(d), p(d)}(t, d)\right|^{2}$ is the channel gain between UE $u$ and pico BS $p$.

It is obvious that the SINR threshold $\gamma_{\text {thresh }}$ defines the discovery region of a UE in the proposed D2D discovery method. Different threshold values are examined in the next section to demonstrate the effect of it on the D2D discovery performance.

To further improve the D2D discovery performance, we consider time-domain ICIC within the heterogeneous cellular network. Macro and pico BSs perform ICIC by ABSs as proposed in [42]. Within ABSs either the macro BSs or the pico BSs mute their transmission. Based on a selected ABS ratio $R_{\mathrm{ABS}}$, BSs do not transmit on certain subframes out of $\left[1, \ldots, N_{\mathrm{T}}\right]$ subframes, i.e., they cause no interference on these subframes. An illustration of the muting frame structure is depicted in Fig. 3.3(a) for different ABS ratios. Fig. 3.3(b) shows the DR distribution over $N_{\mathrm{T}}$ subframes. Considering a decentralized approach in which the UEs are not informed about the ABSs ratio $R_{\mathrm{ABS}}$, UEs select DRs based on the minimum received power according to the proposed metric in equation (3.5). Subframes with ABS will be 


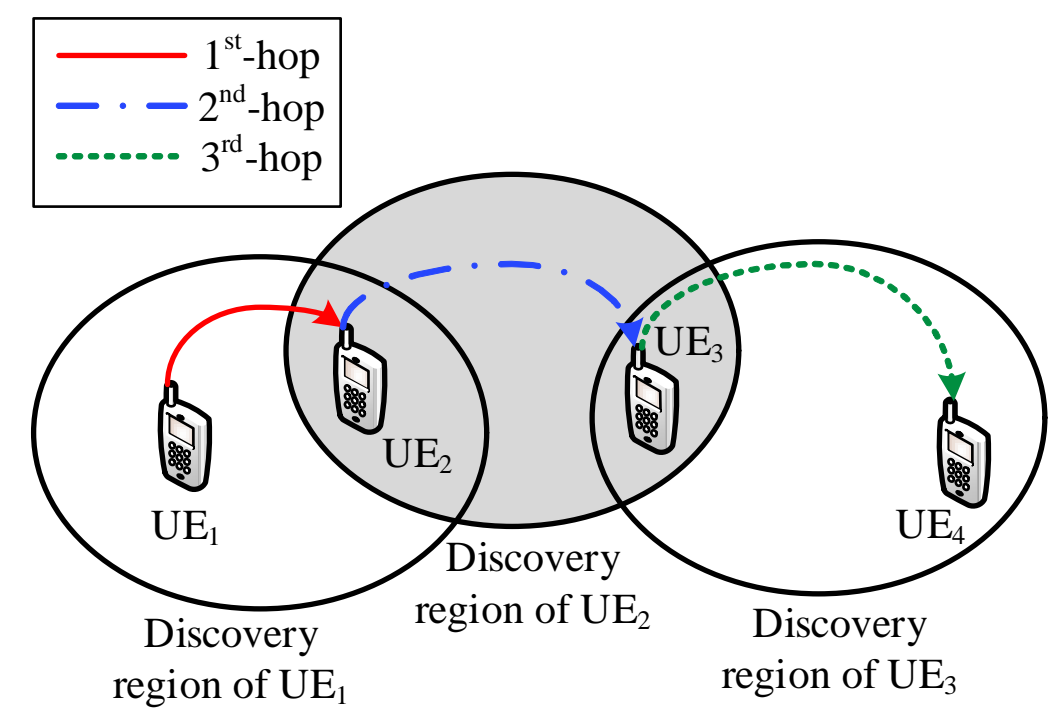

Figure 3.4: Illustration of multi-hop discovery of far away UEs.

preferred due to lower interference and can discover more UEs and more simultaneous DR transmissions by UEs. This enables UEs in close proximity to a macro/pico BS to perform D2D discovery in ABSs by selecting their DR in these subframes. However, muting subframes will lead to the case that many UEs select DRs in the ABSs due to the lower received power on these subframes. This will cause more interference by the UEs that transmit their peer discovery information on the same DRs so that consequently less UEs will be discovered. Hence, there is an optimum ABS ratio and SINR threshold in the proposed ICIC method, which is demonstrated based on system level simulations in Section 3.5.

Additionally, multi-hop discovery is considered. By using multi-hop, UEs can discover each other even when they are located in different discovery regions. As depicted in Fig. 3.4 we consider a multi-hop approach in which $\mathrm{UE}_{1}$ can discover in 1-hop $\mathrm{UE}_{2}$ in its own discovery region. In case of 2-hops $\mathrm{UE}_{1}$ can discover $\mathrm{UE}_{3}$ which is in $\mathrm{UE}_{2}$ 's discovery region over $\mathrm{UE}_{2}$. Using multi-hop D2D discovery a UE's discovery region is virtually expanded. 


\subsection{System Model Simulation Results on D2D Discovery}

This section presents system level simulation results for device discovery based on the 3GPP simulation and layout assumptions for HetNets and D2D discovery [25, 42,43]. It is assumed that the considered HetNet consists of $M=1$ macrocell, $K=2$ uniformly positioned picocells per macro sector, and $U=450$ (150 per macro sector) UEs which are uniformly dropped based on layout option 5 assumptions in [48]. We focus on this layout option since it is mandatory especially for public safety scenarios. Throughout the simulations we assume that $N_{\mathrm{F}}=N_{\mathrm{RB}}$ (unless stated otherwise), i.e. all RGs in time-and frequency domain are available for discovery, whereby DRs are selected based on the PDRID selection algorithm. Further details about system level simulation parameters are provided in Table 3.1. System level simulations have been performed for various random scenarios, whereby we consider the same random layouts and channels for each algorithm to enable a fair comparison.

Fig. 3.5 depicts the cumulative distribution function (CDF) of the number of discovered UEs for the two D2D discovery approaches random and smart PDRID selection for different number of subframes in case of dedicated channel D2D and regular UEs for an SINR threshold $\gamma_{\text {thresh. }}$ It can be observed that the smart PDRID approach yields in all cases a better performance than the random PDRID selection approach.

To demonstrate the performance of the proposed methods in case of co-channel deployments and ABS based ICIC, we evaluate the performance of the average number of discovered UEs vs $N_{\mathrm{T}}$ in Fig. 3.6 and Fig. 3.7 for macro cell based ICIC and pico cell based ICIC, respectively. The different ABS ratios $R_{\mathrm{ABS}}$ are the iteration step size of subframes over which the BSs mute, i.e. $R_{\mathrm{ABS}}=1$ means that the BS mutes every second subframe and $R_{\mathrm{ABS}}=0$ means that the BS does not mute (see Fig. 3.3(a)). 
Table 3.1: Simulation parameters for D2D discovery.

\begin{tabular}{|l|l|}
\hline Parameter & Value \\
\hline Cellular layout & $\begin{array}{l}\text { Hexagonal grid, } \\
3 \text { sectors per cell, reuse 1 }\end{array}$ \\
\hline Carrier frequency & $700 \mathrm{MHz}$ \\
\hline System bandwidth & $10 \mathrm{MHz}$ \\
\hline Subframe duration & $1 \mathrm{~ms}$ \\
\hline Number of RGs & $N_{\mathrm{F}}=50$ \\
\hline Number of macrocells/picocells & $M=1 / K=6$ \\
\hline $\begin{array}{l}\text { Maximum transmit power } \\
p_{\mathrm{tx}}^{m}, p_{\mathrm{tx}}^{k}, p_{\mathrm{tx}}^{u}\end{array}$ & $46 \mathrm{dBm}, 30 \mathrm{dBm}, 23 \mathrm{dBm}$ \\
\hline Number of UEs per sector & $150(U=450)$ \\
\hline UE2UE path loss model & Winner channel model (see [49]) \\
\hline UE2UE shadowing correlation & Spatially correlated \\
\hline BS2UE path loss model & According to table B.1.2.1-1 in [42] \\
\hline BS2UE shadowing correlation & Time correlated \\
\hline Shadowing standard deviation & $\begin{array}{l}\text { Independent and identically distributed } \\
\text { (iid) }\end{array}$ \\
\hline Minimum distance UE2UE & $3 \mathrm{~m}$ \\
\hline Thermal noise density & $-174 \mathrm{dBm}$ \\
\hline
\end{tabular}

It can be observed that there is no gain in case of macro ICIC. This is due to the fact that the macro BS transmit power is high and UEs select their discovery resources mainly on subframes on which the macro BS mutes, which leads to a high UE2UE interference.

In case of pico ICIC, we observe high performance improvements for the smart PDRID selection. This is because of the lower transmit power of pico BSs, which does not lead to overloaded ABSs. It can be seen that $N_{\mathrm{T}}=8$ yields the best performance. Therefore, the following results will focus on performance evaluations for this number of discovery subframes. However, it has to be pointed out that $N_{\mathrm{T}}=8$ may lead to delays in UE discovery. 


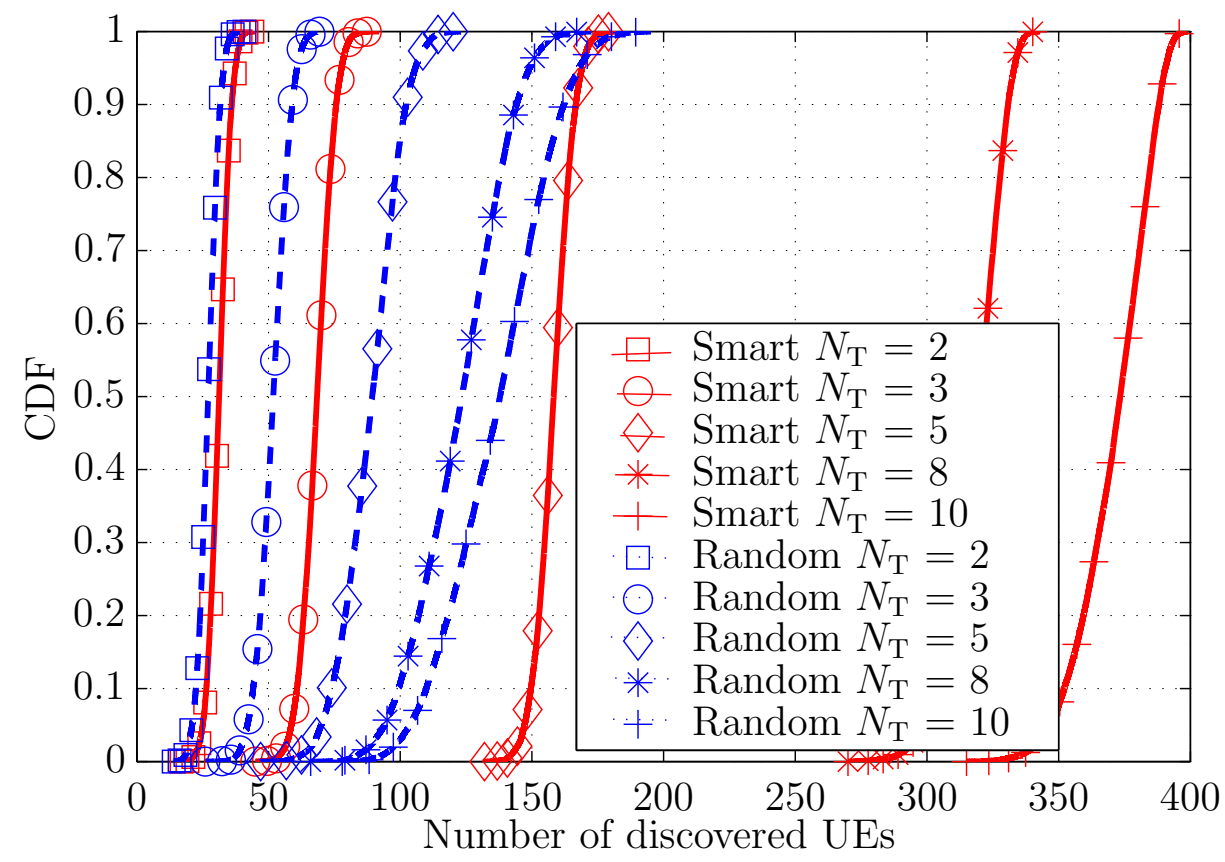

Figure 3.5: CDF of number of discovered UEs in the absence of ICIC for random and smart PDRID selection, and $\gamma_{\text {thresh }}=8 \mathrm{~dB}$.

Fig. 3.8 and Fig. 3.9 depict the average number of discovered UEs vs SINR threshold $\gamma_{\text {thresh }}$ for the smart PDRID selection algorithm for different ABS ratios. In case of pico ICIC the average number of discovered UEs is increasing for increasing number of ABSs while it is almost the same in case of macro ICIC. The wider a UE's discovery range (the smaller $\gamma_{\text {thresh }}$ ) is, the more UEs can be discovered. Reducing the value of $\gamma_{\text {thresh }}$, even when that would help to increase the number of discovered UEs, is not a practical criteria, because that would sacrifice the channel conditions of the D2D links.

To further improve the number of discovered UEs, we performed multi-hop based D2D discovery. The effect of interference from BSs on the discovery performance and the benefit of using multi-hop discovery in the absence of ICIC for 1-hop, 2-hop and 3-hop can be found in Fig. 3.10. In general, the use of multi-hop will increase the discovery region size for all UEs without sacrificing channel quality. 


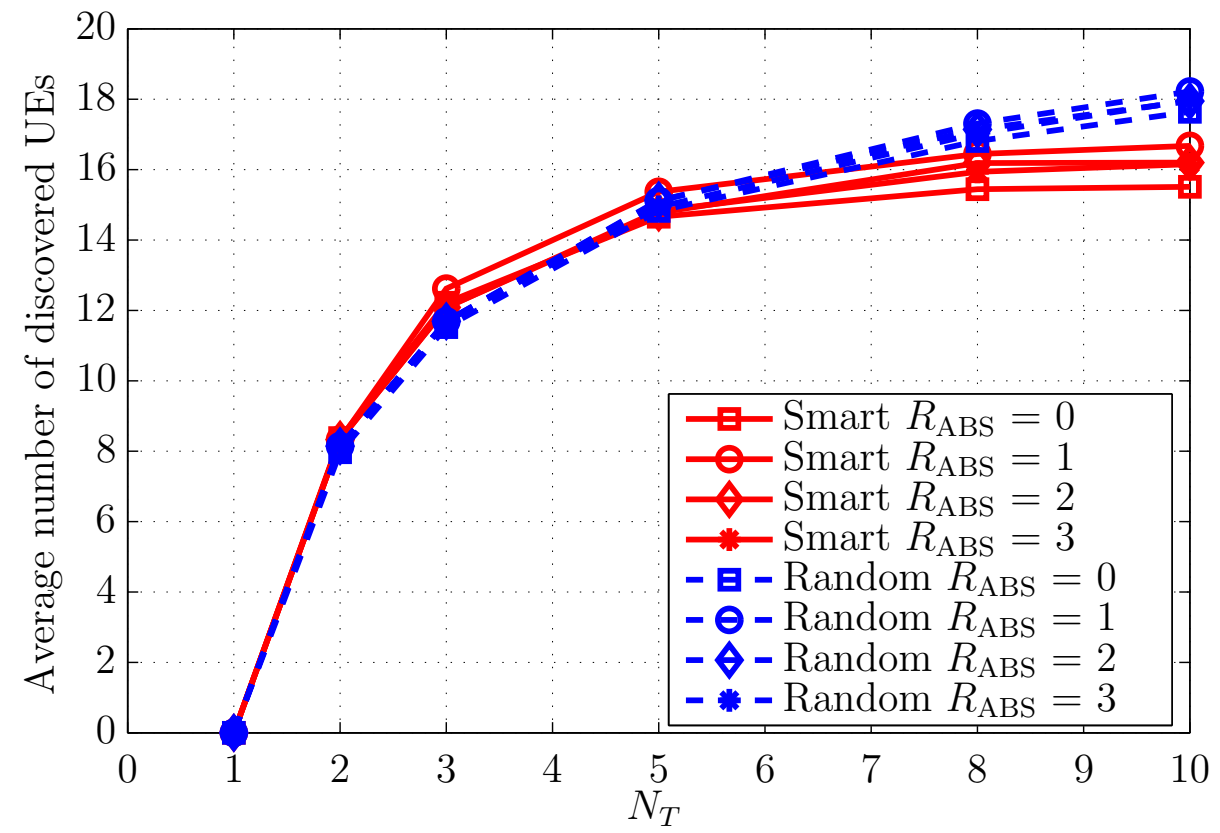

Figure 3.6: Average number of discovered UEs vs $N_{T}$ for various ABS ratios in case of macro ICIC and $\gamma_{\text {thresh }}=8 \mathrm{~dB}$.

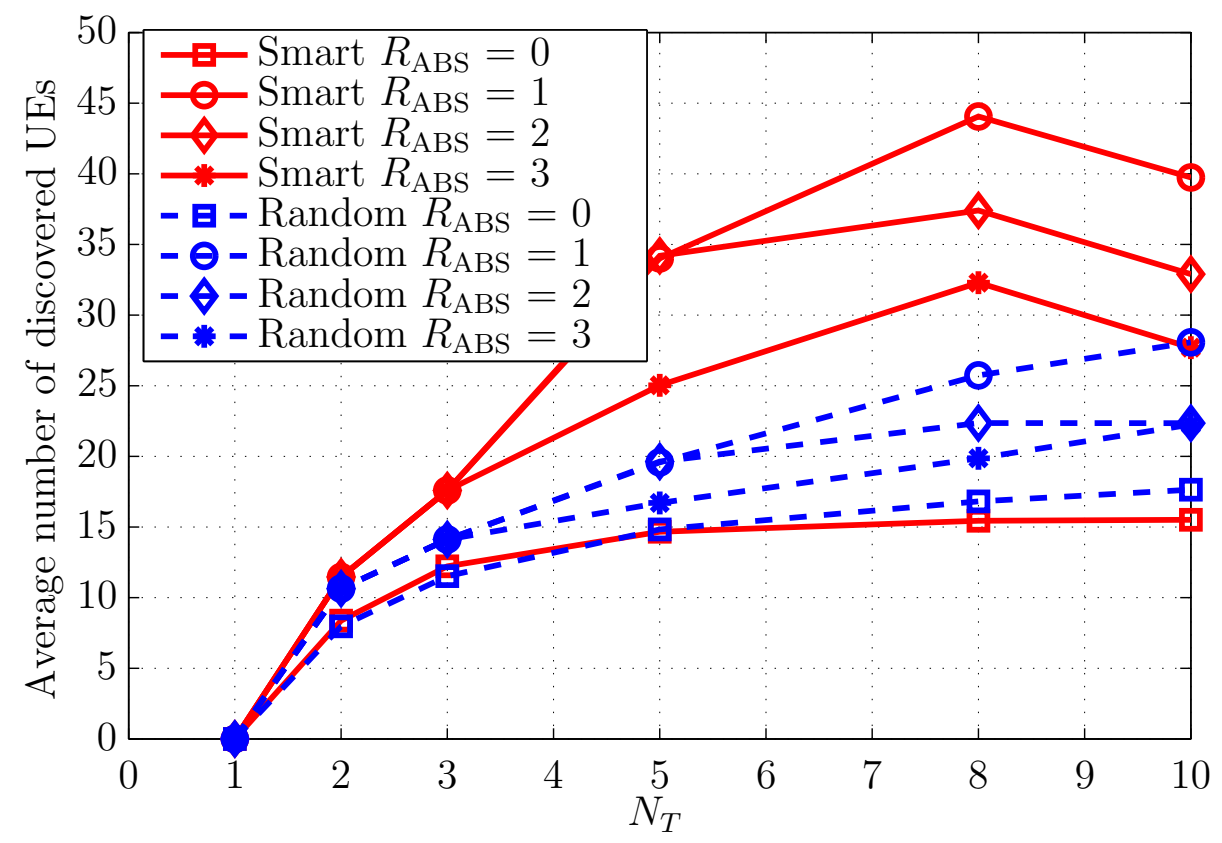

Figure 3.7: Average number of discovered UEs vs $N_{T}$ for various ABS ratios in case of pico ICIC and $\gamma_{\text {thresh }}=8 \mathrm{~dB}$. 


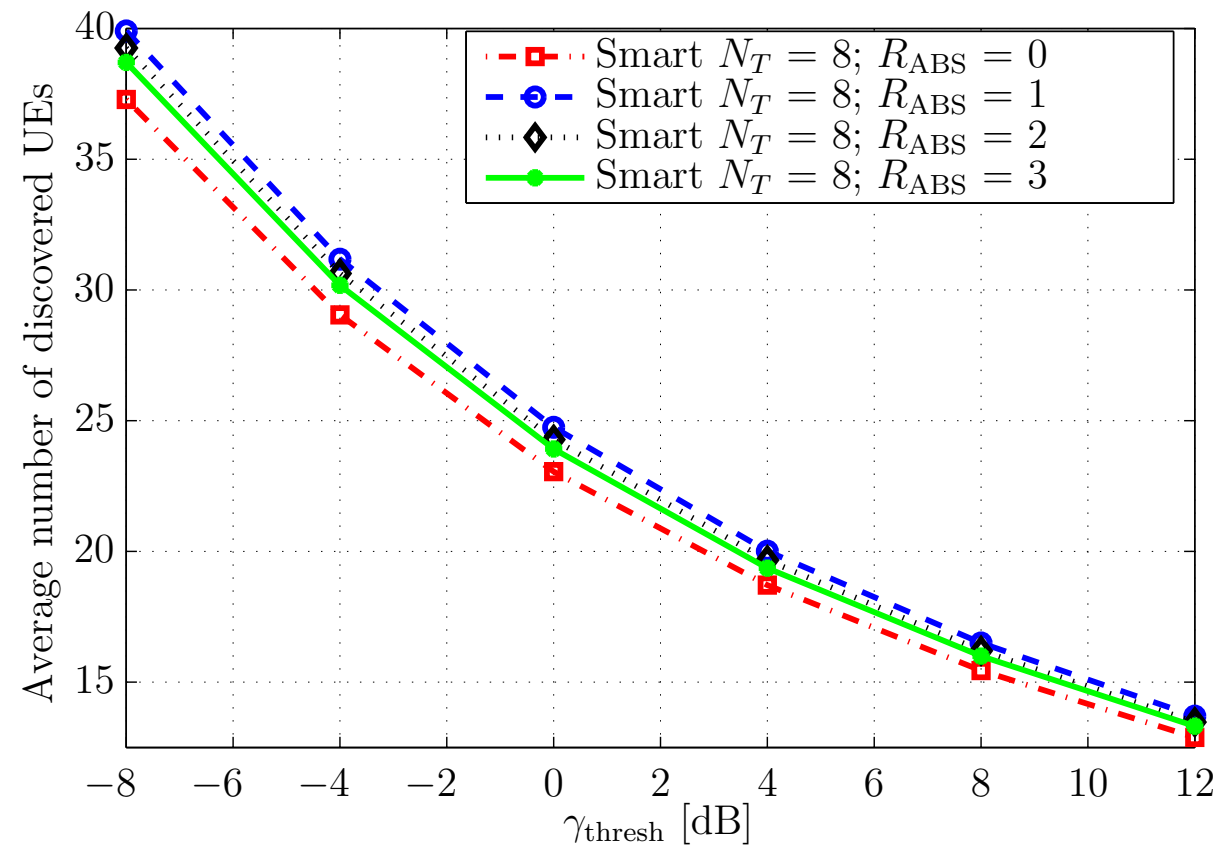

Figure 3.8: Average number of discovered UEs vs $\gamma_{\text {thresh }}$ for various ABS ratios in case of macro ICIC.

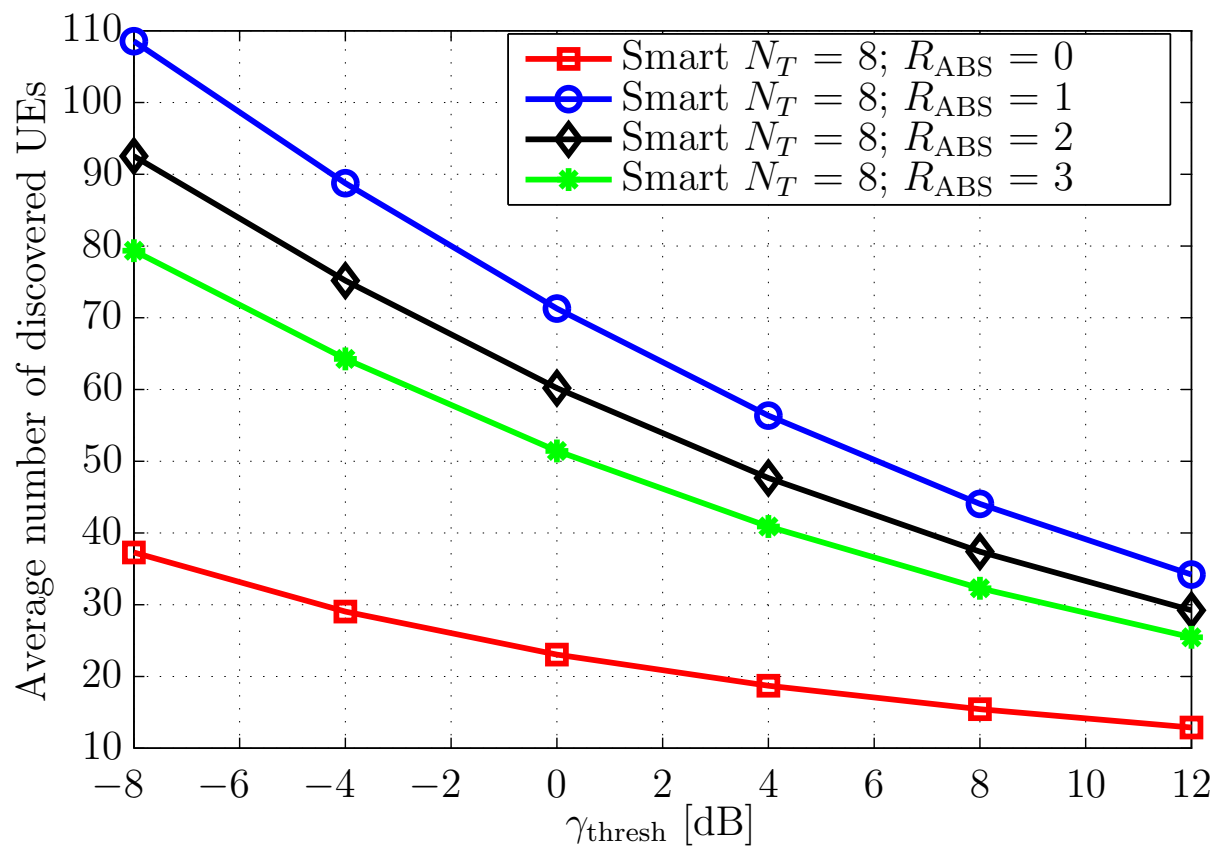

Figure 3.9: Average number of discovered UEs vs $\gamma_{\text {thresh }}$ for various ABS ratios in case of pico ICIC. 


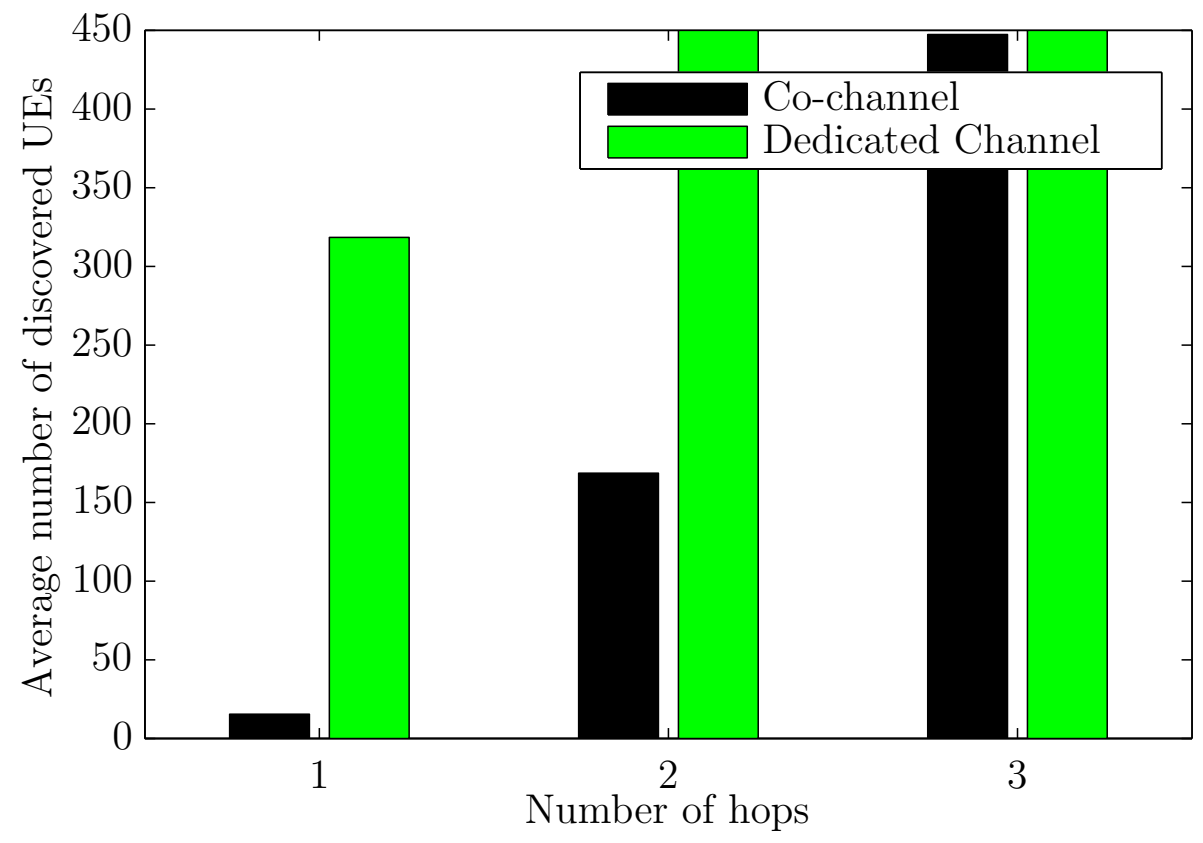

Figure 3.10: Average number of discovered UEs for multi-hop in the absence of ICIC.

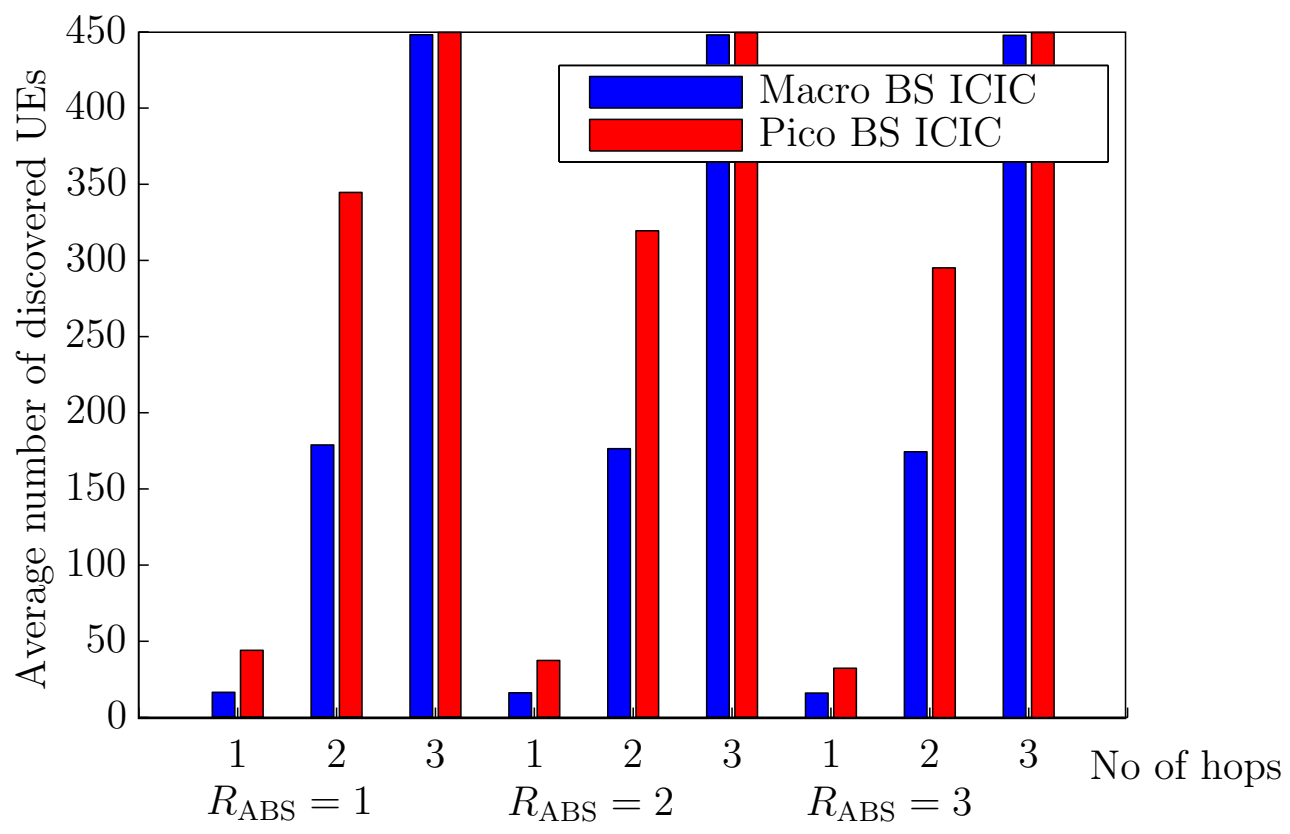

Figure 3.11: Average number of discovered UEs for multi-hop discovery and different ABS ratios. 
It is observed that dedicated channel deployment of D2D UEs results in significantly larger number of UEs to be discovered. On the other hand, even with cochannel deployments, UEs are able to discover all the other UEs after three hops.

Fig. 3.11 investigates the performance of multi-hop D2D discovery approach in co-channel deployments and with different ABS rates at the macrocell and picocells. Three different $\mathrm{ABS}$ ratios are considered: $R_{\mathrm{ABS}}=1, R_{\mathrm{ABS}}=2$, and $R_{\mathrm{ABS}}=3$. Comparing with Fig. 3.10, use of ICIC leads to larger number of discovered UEs, especially when implemented at picocells. The average number of discovered UEs increases with the number of hops, but also depends on the different ABS ratios in both macro and pico BSs. In all cases, all UEs are discovered after 3 hops. 


\section{CHAPTER 4 \\ D2D COMMUNICATIONS IN PUBLIC SAFETY PARTIAL COVERAGE SCENARIOS}

In this chapter, we study the D2D communication mechanisms and the use of multi-hop communications for enhancing data capacity, EE and SE in public safety partial coverage scenarios. As we mentioned in Chapter 1, MBSs are vulnerable to large-scale disasters like earthquakes, hurricanes, tornadoes and terrorist attacks. Emergency situations can generate partial coverage scenarios and, since emergency communications become critical for public safety, it is important to overcome this situation by, for example, extending the coverage area of those MBSs that remain active. D2D communications and the use of in-coverage UEs as relay, might constitute a viable solution to this problem.

\subsection{Introduction to Device-to-Device Communication Pro- cess}

Contrary to D2D discovery signal, which contains only few bits, D2D communication data often involves data streaming, local gaming etc, and thus can be much heavier. In addition, the total traffic varies a lot in time. Therefore, dedicated resources use is inefficient for D2D data communications. Overlapping resources with cellular transmission is highly desired in order to make better use of the spectrum.

The key problem in overlapping resource use is the interference management. Interference from D2D to cellular transmissions needs to be strictly controlled by the

network in order to protect cellular transmission when D2D UEs are in coverage or in partial coverage. 
In general, to coordinate D2D communication with cellular network in order to reduce interference and to achieve efficient resource reuse, the following techniques can be used [38]:

- Resource allocation: Resources can be orthogonal between D2D and cellular transmissions and/or among D2D transmissions in order to reduce intra-cell interference. However, the spectrum might be underutilized, which leads to spectral efficiency problems. Intra-cell resource reuse based on location information and/or SINR estimation are more promising in the optimal use of resources.

- Power control: Taking advantage of the proximity of D2D UEs, power control is another element that can be used to manage interference. Reducing the transmission power of closed-enough D2D UEs might reduce the interference with cellular network and/or other D2D communications while guaranteeing the desired QoS.

- Hybrid Automatic Repeat Request (HARQ): Can enhance D2D performance in spatial reuse scheme by simple repetition of D2D transmissions in multiple subframes. This technique is specially beneficial during D2D transmissions out of network coverage, where the interference can be severe, and required transmission range of public safety applications can be long.

- Mode Selection: Mode selections allow a soft switch between D2D mode and conventional UL/DL mode communications in order to achieve efficient resource use.

\subsection{D2D Communications Routing Algorithms}

Once the discovery process defines possible D2D networks, the use of multi-hop techniques will guarantee the communication between source and destination by using 
intermediate UEs as wireless relays. Multi-hop routing is a key factor in D2D communications since, as mentioned before, several metrics related with the multi-hop algorithm can affect the performance of the D2D communication [50].

Multi-hop routing has been studied extensively for wireless communications in the past and several algorithms have been proposed for supporting D2D communications [19-22, 51,52]. However, depending on their specific characteristics, these algorithms may or may not be applied to specific communication scenarios.

In general, the main challenge for routing in D2D networks includes three different layers. First, the transmit data constraints due the battery life limitation in UEs impact the route selection significantly as well as the sustainable throughput. Second, the geometric information of the D2D network, interference constraints and the D2D rate requirements (based on the proximity services supported) need to jointly be considered to optimize the routing strategies. Third, the computational complexity of D2D algorithms has to be taken in consideration as this significantly impacts the energy efficiency and fast data forwarding requirements [22]. The total number of hops, hop distance, total supported communication capacity, energy efficiency, and probability of routing success are some examples of metrics that we can use to compare different routing algorithms. Some of these routing algorithms also considers the angle of hop for selecting the next relay [52].

Some examples of multi-hop routing algorithms suitable to be used for LTE D2D communication are as follows:

- Shortest-Path-Routing (SPR) Algorithm: In SPR, each D2D UE knows its location by using Global Position System (GPS) or other wireless localization means. The SPR algorithm for a generic UE pair $(s, d)$ first requests communication from source $\mathrm{UE}_{s}$ to destination $\mathrm{UE}_{\mathbf{d}}$. Then, source UEs sends relay request to all neighboring UEs in its communication range $R$. After receiving 
feedback of potential relay UEs available, source $\mathrm{UE}_{s}$ sends data packet to the relay $\mathrm{UE}$ that is the closest to the destination $\mathrm{UE}_{d}[19]$.

- Interference-Aware-Routing (IAR) Algorithm: The idea behind IAR is to reduce the interference from MBS to the D2D links [19]. In this case, the multi-hop route attempts to travel along the circumference of an MBS coverage area. This is a modification of SPR algorithm since in the three different stages of IAR, SPR algorithm is used as follows:

1. Escape: Multi-hop from source $\mathrm{UE}_{s}$ to closest boundary UE.

2. Migrate: Multi-hop from the boundary UE to a boundary UE that is closest to the destination $\mathrm{UE}_{d}$.

3. Return: Multi-hop from the boundary UE to the destination $\mathrm{UE}_{d}$.

IAR algorithm increases the path length significantly in comparison with SPR, but the advantages are that the interference from MBS can be reduced considerably due to the increased distance from MBS.

- FlashLinQ and Two-Hop FlashLinQ: Introduced by Qualcomm, this technique is based in a new single-tone (high-energy) analog signal. FlashLinQ (FQL) enables D2D discover in areas over $1 \mathrm{~km}$ radius and requires a smaller number of hops [20]. On the other hand, single-tone signals cannot guarantee the minimum performance of the data transfer through multitone (low-energy) signals. Two-Hop FLQ is a variant of FLQ, proposed to overcome the limitation of FlashLinQ. Instead of one long hop, the new technique proposes a new two-hop communication by the help of an intermediate device.

- Maximum Rate Towards Destination (MR-D): This is a modification of SPR algorithm. MR-D algorithm tries to reach the destination UE in a D2D link by using the least amount of hops. This would reduce the delay and complexity, 
while guaranteeing the minimum sustainable rate towards the destination for the required QoS [22].

- Farthest Neighbor (FN) Routing Algorithm: The FN algorithm is a typical routing technique used in interference constrained environments. The idea behind FN is to reach the node, as far as possible from the current position, on condition that the rate requirements for the $\mathrm{D} 2 \mathrm{D}$ communications can be satisfied [22].

\subsection{D2D System Level Simulation Environment for Partial Coverage Scenarios}

The 3GPP based D2D system level simulator studied in Chapter 3 has been modified to create partial coverage scenario conditions.

It is assumed that the considered network consists of $M=4$ macrocells, and $U=240$ (20 per macro sector) UEs which are uniformly dropped based on layout option 5 assumptions in [48]. We focus on this layout option since it is mandatory especially for public safety scenarios. Further details about system level simulation parameters are provided in Table 4.1. For simplification purposes, picocells are not considered.

We have defined a public safety scenario with a total of four MBSs. The two MBSs located in the upper region of the model are transmitting at full power, while the two MBSs located in the lower region of the model are muted, defining the noncovered zone. The total deployment of the four macro base stations creates the partial coverage scenario.

For communication purposes, we consider a scenario of D2D communications underlying a cellular network where a far-away $U E_{1}$ intends to communicate with the 
Table 4.1: Simulation parameters for D2D communications.

\begin{tabular}{|l|l|}
\hline Parameter & Value \\
\hline Cellular layout & $\begin{array}{l}\text { Hexagonal grid, } \\
\text { 3 sectors per cell, reuse 1 }\end{array}$ \\
\hline Carrier frequency & $700 \mathrm{MHz}$ \\
\hline System bandwidth & $10 \mathrm{MHz}$ \\
\hline Subframe duration & $1 \mathrm{~ms}$ \\
\hline Number of RGs & $N_{\mathrm{F}}=50$ \\
\hline Number of macrocells & $M=4$ \\
\hline Number of active macrocells & $M=2$ \\
\hline $\begin{array}{l}\text { Maximum transmit power } \\
p_{\text {tx }}^{m}, p_{\text {tx }}^{u}\end{array}$ & $46 \mathrm{dBm}, 23 \mathrm{dBm}$ \\
\hline Communications scenario & Uplink \\
\hline Number of UEs per sector & $20(U=240)$ \\
\hline UE2UE path loss model & Winner channel model (see [49]) \\
\hline BS2UE path loss model & According to table B.1.2.1-1 in [42] \\
\hline Minimum distance UE2UE & $3 \mathrm{~m}$ \\
\hline Thermal noise density & $-174 \mathrm{dBm}$ \\
\hline
\end{tabular}

active MBS which is closer to its location. $U E_{1}$ is located in a non-covered area and will try to communicate with the base station $B S_{1}$ directly and by using other UEs as relay. Four different routes have been predefined: direct communication with the base station (1-hop), communication with the base station by using 1 UE as relay (2-hops), communication with the base station by using 2 UEs as relays (3-hops) and finally, communication with the base station by using 3 different UEs as relays (4hops). A second multi-hop route is defined where a far-away $U E_{2}$ will try to connect with the active base station $B S_{2}$ directly and using 1,2 and 3 hops relays.

Since every communication hop is occurring in different time frames $T_{r, l, h}$, where $r$ defines the route number, $l$ defines the link number for a specific route and $h$ defines the hop number within a specific link, all relays can be reused for different routes and links numbers (see Fig. 4.1). 

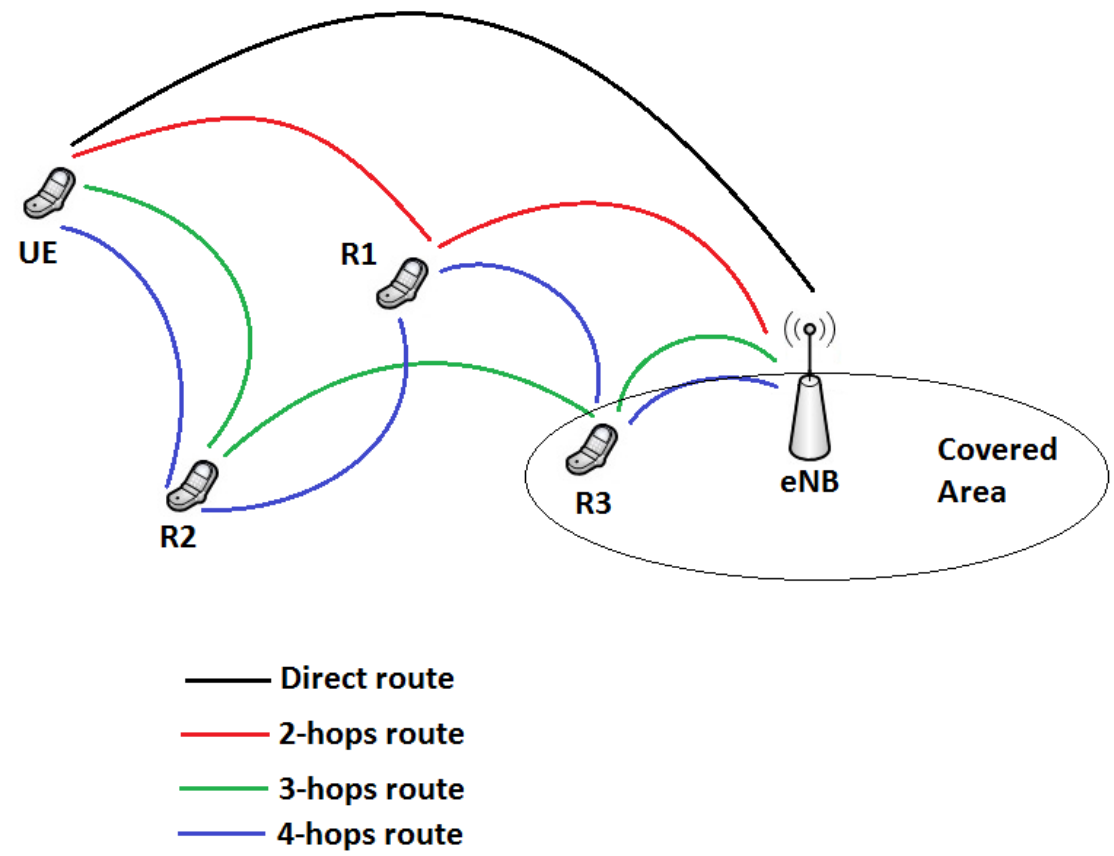

Figure 4.1: Multi-hop routes model used for communication purposes in public safety partial coverage scenario.

It is assumed that every communication link $L_{r, l}$ is composed by $H$ hops $h_{r, l, h}$. Every hop $h_{r, l, h}$ from the same route occupies a different $T_{r, l, h}$ LTE resource hence, interference is only possible within the same $h_{r, l, h}$ hop duration from different routes (see Fig. 4.2). Other UEs' transmissions are assumed to be orthogonal to our model. In this specific case, route 1 is considered as useful communication and route 2 is considered as interference to route 1.

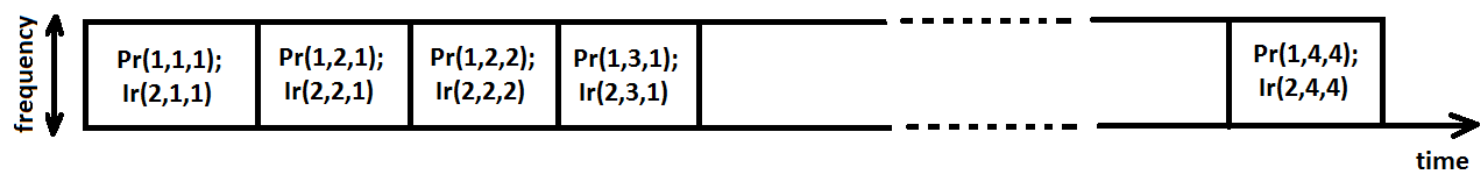

Figure 4.2: Full multi-hop routes scheduling in LTE time frames for D2D communications received power $P_{r}(r, l, h)$ and interference received power $I_{r}(r, l, h)$. 


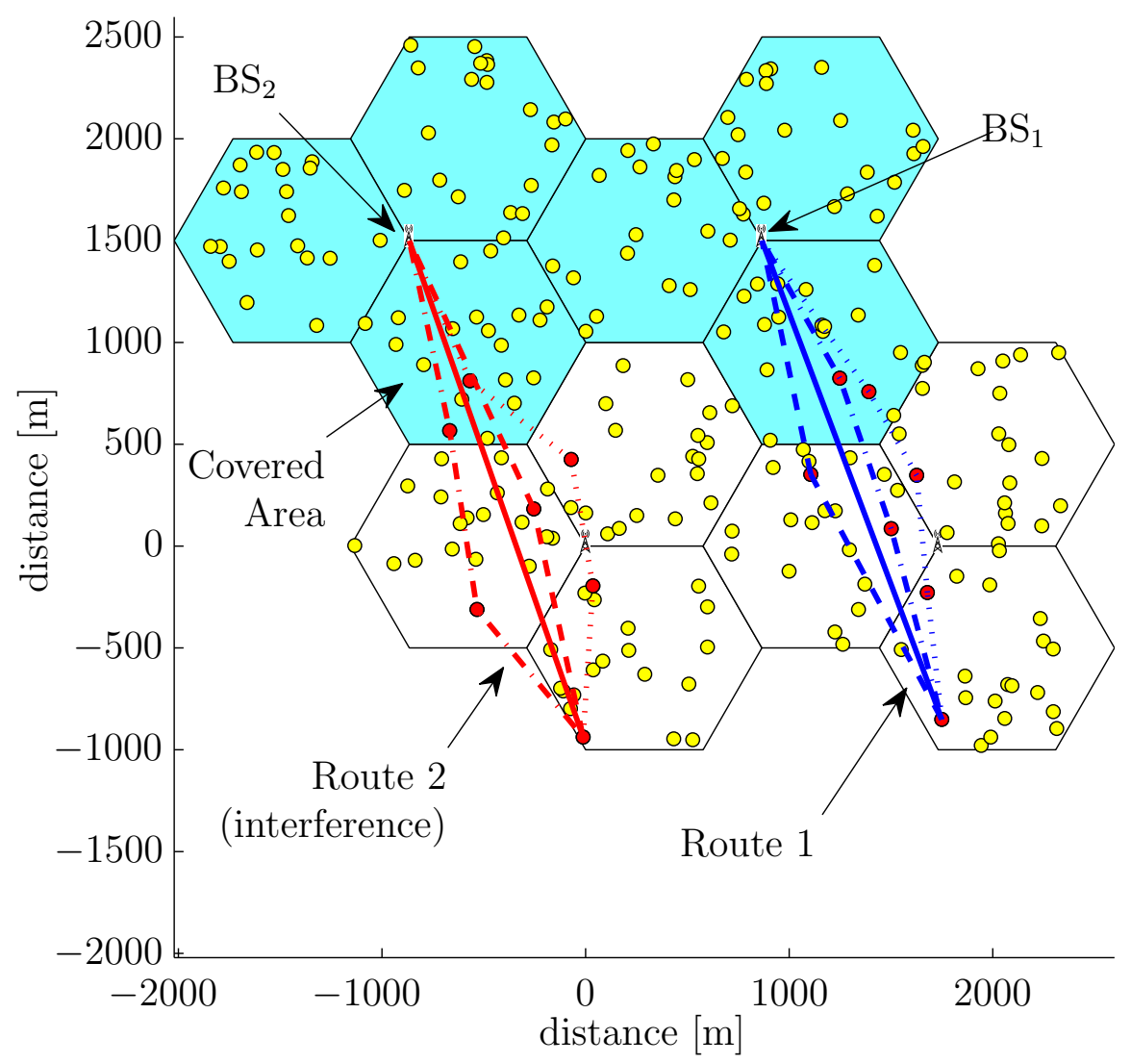

Figure 4.3: 3GPP public safety partial coverage scenario [3] and defined D2D routes for evaluation purposes.

At the end, every route will have four links in total, one direct link from $U E_{1}$ to $B S_{1}$ and $U E_{2}$ to $B S_{2}$ respectively, and 3 multi-hop links (see Fig. 4.3).

\subsubsection{Energy Efficiency and Spectral Efficiency for Multi-hop D2D Communications}

Since every communication path within a route is independent and occurs in different time slots, we can define capacity $C_{1, l}$ for route 1 as [53]:

$$
C_{1, l}=\sum_{i=1}^{N} B \log _{2}\left(1+S I N R_{l, i}\right)
$$


with $S I N R_{l, i}$ representing the signal-to-interference-plus-noise-ratio for every hop $i$ within the link $l$.

Since noise can be considered negligible, $S I N R_{l, i}$ becomes signal-to-interferenceratio $S I R_{l, i}$ calculated by:

$$
S I R_{l, i}=\frac{P r_{l, i}}{I r_{l, i}}
$$

with $I_{r_{l, j}}$ received power of the interference from route 2 in hop $i$ and link $l$.

At the end, total capacity for all multi-hop communications in route 1, will define the energy efficiency (EE) and spectral efficiency (SE) performance of multi-hop D2D communications in our model.

The overall instantaneous transmission vector for energy efficiency $E E(1, l)$ comprised by the EE elements from every link $L_{1, l}$ is defined by:

$$
E E_{1, l}=\frac{C_{1, l}}{H p_{\mathrm{tx}}^{u}}
$$

where $H$ represents the number of hops on every link $L_{1, l}$ and $p_{\mathrm{tx}}^{u}$ the maximum transmission power of the UE.

The same way, we can define SE vector for route 1 as:

$$
S E_{1, l}=\frac{C_{1, l}}{B}
$$

\subsection{System Model Simulation Results on D2D Communica- tions}

In this section, we are presenting simulation results that demonstrate the benefits of using D2D with multi-hop communications for extending the coverage area of an MBS in partial coverage scenarios during public safety communications. Far-awaynot-covered UEs can benefit from using other UEs as communication relay to reach 
the active MBSs. D2D communications and multi-hop may increase the total system capacity while improving energy and spectral efficiency. Also, the addition of UEs in $\mathrm{D} 2 \mathrm{D}$ communication increases the total number of users being served by the base station, which is critical is public safety scenarios.

In Fig. 4.4, we are evaluating the energy efficiency performance versus number of hops. Simulation results demonstrate that with the increment of number of hops, EE increases, for the same UE transmission power and bandwidth utilization. The use of UEs as relay increases the system capacity due to shorter communication paths which led to better channel conditions. Also, allow for more efficient use of the available bandwidth in public safety domain, when compared with the case of direct communication with the MBS (1-hop).

Fig. 4.5 shows the performance of energy efficiency vs spectral efficiency, for different cases of multi-hop. In both cases, diminishing returns can be observed while incrementing the number of hops for EE performance, this is due the fact that, for higher number of hops, even when the channel conditions are expected to improve, there are some limitations due higher aggregate interference effects.

Another important metric would result after evaluating EE and SE performance while reducing the UE transmission power for different numbers of hops. In public safety communications, reducing the UE transmission power is vital, since that will contribute in extending the UE battery life in situations where power outages can be present by using UE power more efficiently. Also, reducing UE transmission power will reduce the interference of $\mathrm{D} 2 \mathrm{D}$ to cellular network along with the interference with other D2D communications. 


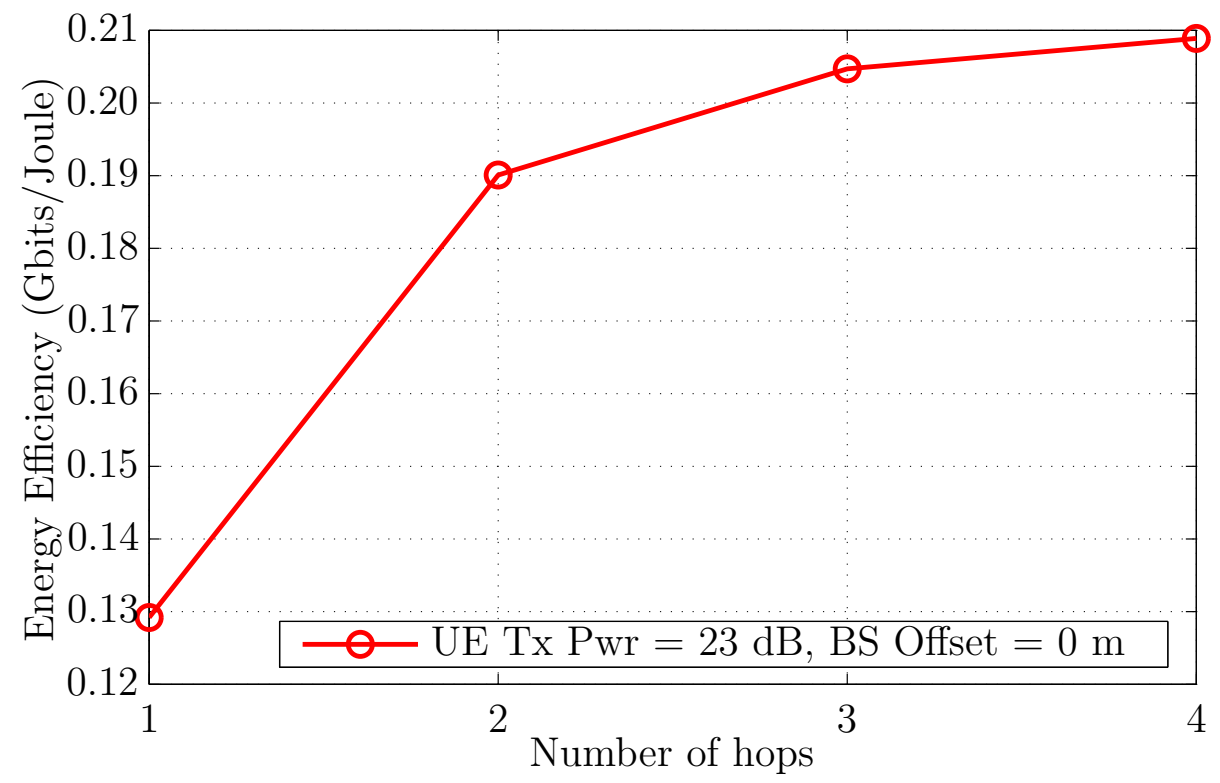

Figure 4.4: Energy efficiency vs number of hops for maximum UE transmission power.

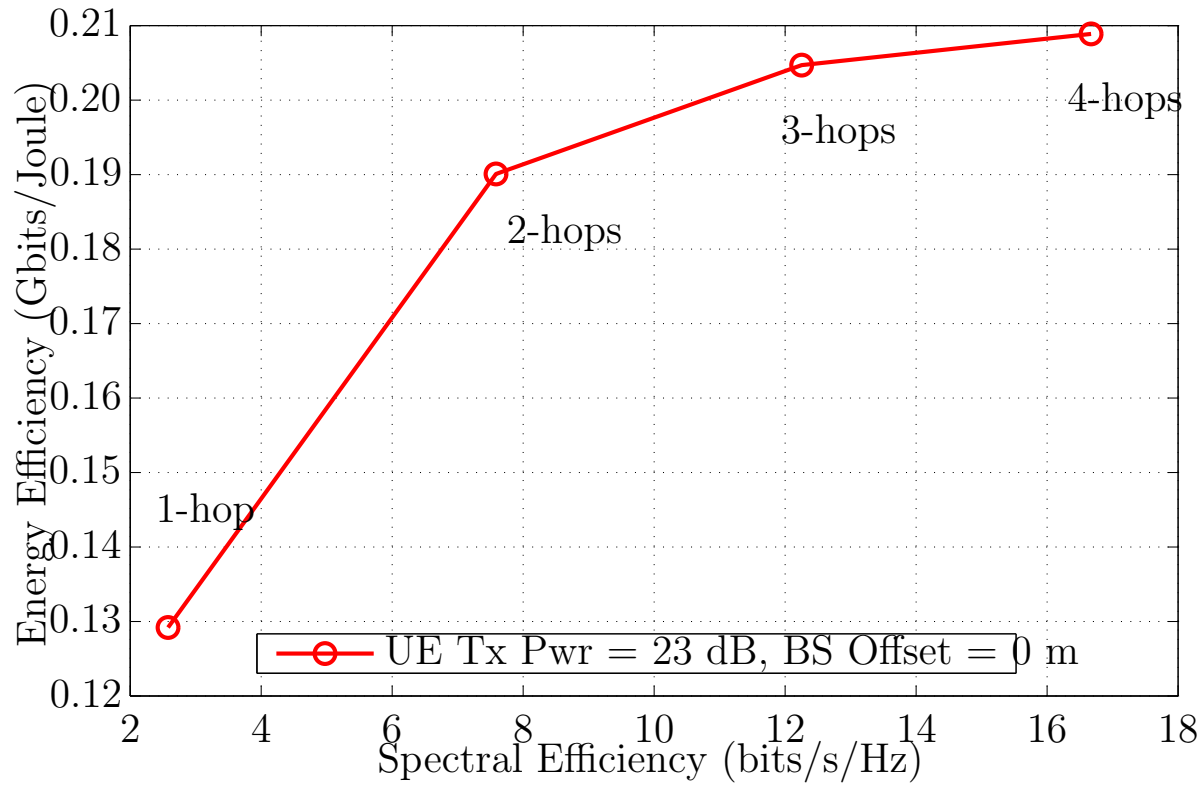

Figure 4.5: Energy efficiency vs spectral efficiency for maximum UE transmission power.

Fig. 4.7 demonstrate that the use of shorther D2D links while increasing the number of hops guarantee or, even improve, the QoS while reducing the UE power 
transmission. That will allow a better use of the UE power resources, increasing the energy efficiency. In Fig. 4.8, the performance of energy efficiency versus spectral efficiency is evaluated. Simulation results shown that reducing the UE transmission power will benefit the energy efficiency of the multi-hop D2D transmissions but will not impact spectral efficiency notably.

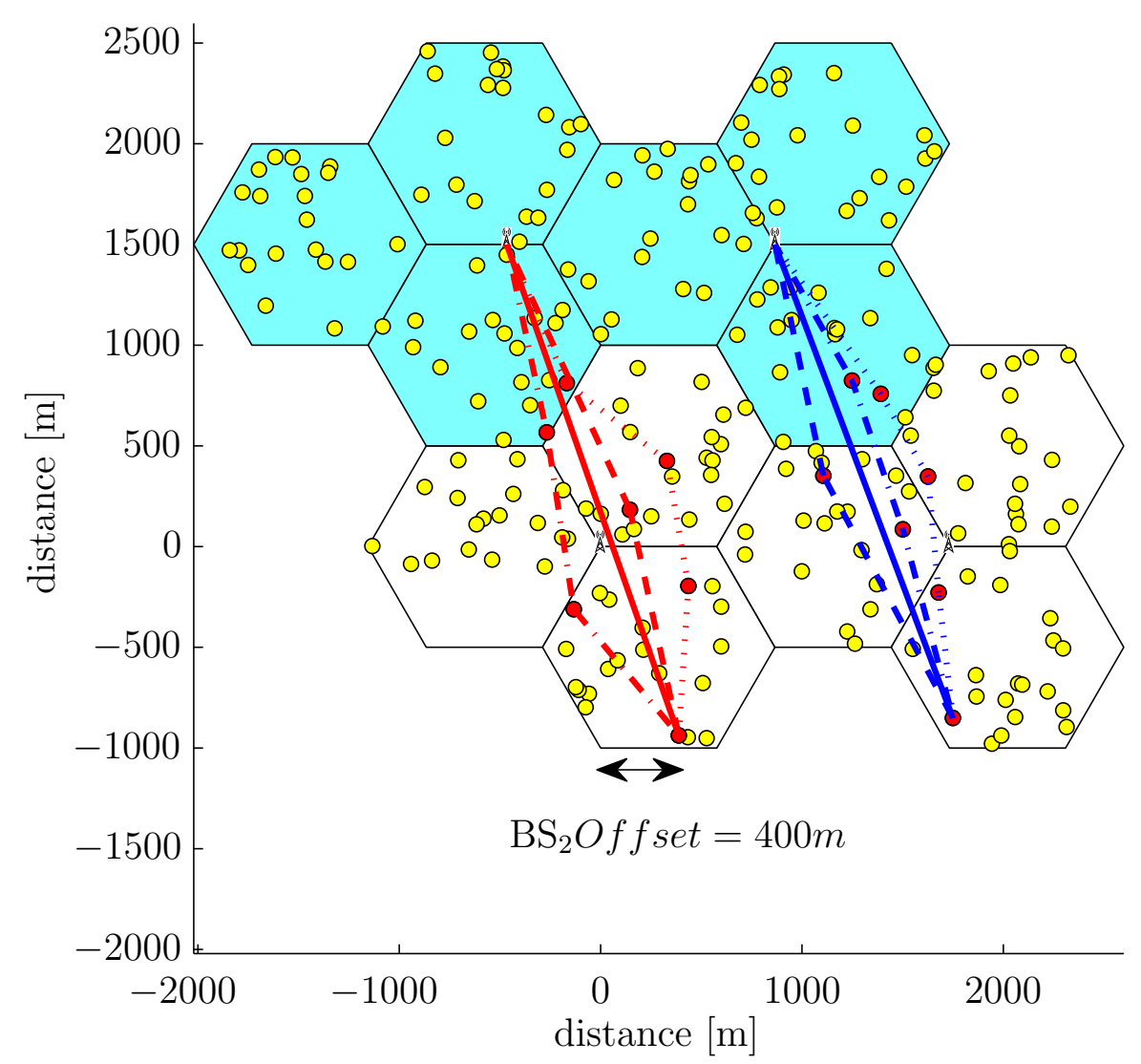

Figure 4.6: System model representation of $B S_{2}$ with $400 \mathrm{~m}$ location offset.

A hypothetical simulation scenario is proposed to evaluate the impact of the interference on the performance of the D2D multi-hop communications. This case moves route 2 towards route 1 by different offsets values, along the $\mathrm{X}$-axis line (see Fig. 4.6). As a consequence, interference over D2D communications in route 1 should increase as result of reducing the horizontal distance between route 1 and route 2 . 
Fig. 4.9 and Fig. 4.10 depict the EE and SE performance versus number of hops for different route 2 positions. Even when D2D communications still benefits from the use of multi-hops, a degradation of $\mathrm{EE}$ and $\mathrm{SE}$ is observed with higher values of route 2 location offsets resulting in higher interference. Worst case scenario is obtained when the interference route 2 is closer to route 1 by $600 \mathrm{~m}$.

Fig. 4.11 demonstrates that, in presence of interference, there exist a tradeoff between EE and SE [54]. For multi-hop communications with more than 3 hops, a little improvement in SE values is observed, while EE suffers a significant degradation. That means, achievable values of EE are limited by the UE transmission power and the interference value. Another obvious conclusion from Fig. 4.11 is that, with higher values of interference, the total performance of $\mathrm{EE}$ and $\mathrm{SE}$ is degraded, but D2D communications still benefits from using multiple UEs as relay because of the better channel conditions achieved with the use of shorter communication links.

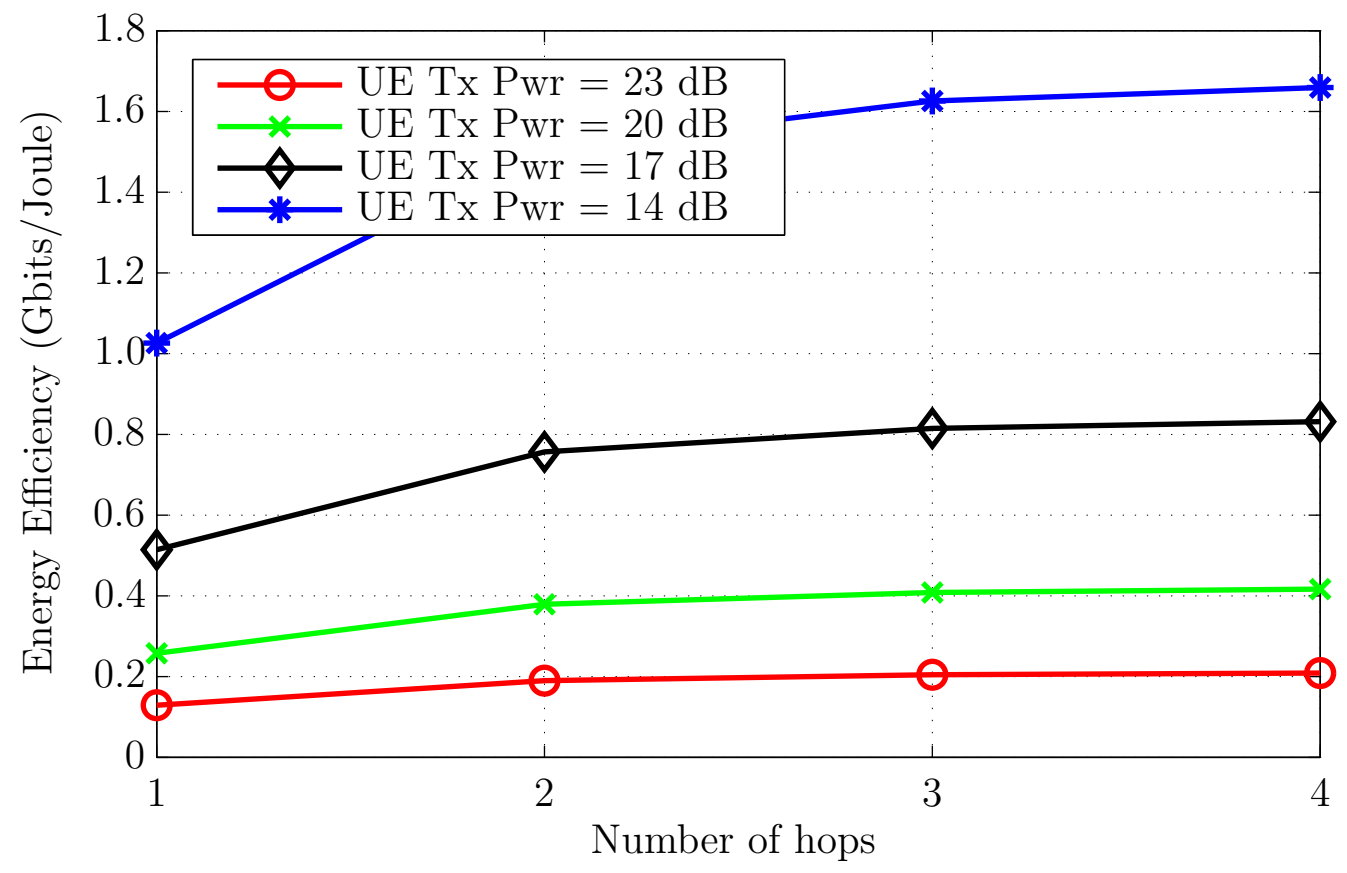

Figure 4.7: Energy efficiency performance vs number of hops while reducing the UE transmission power. 


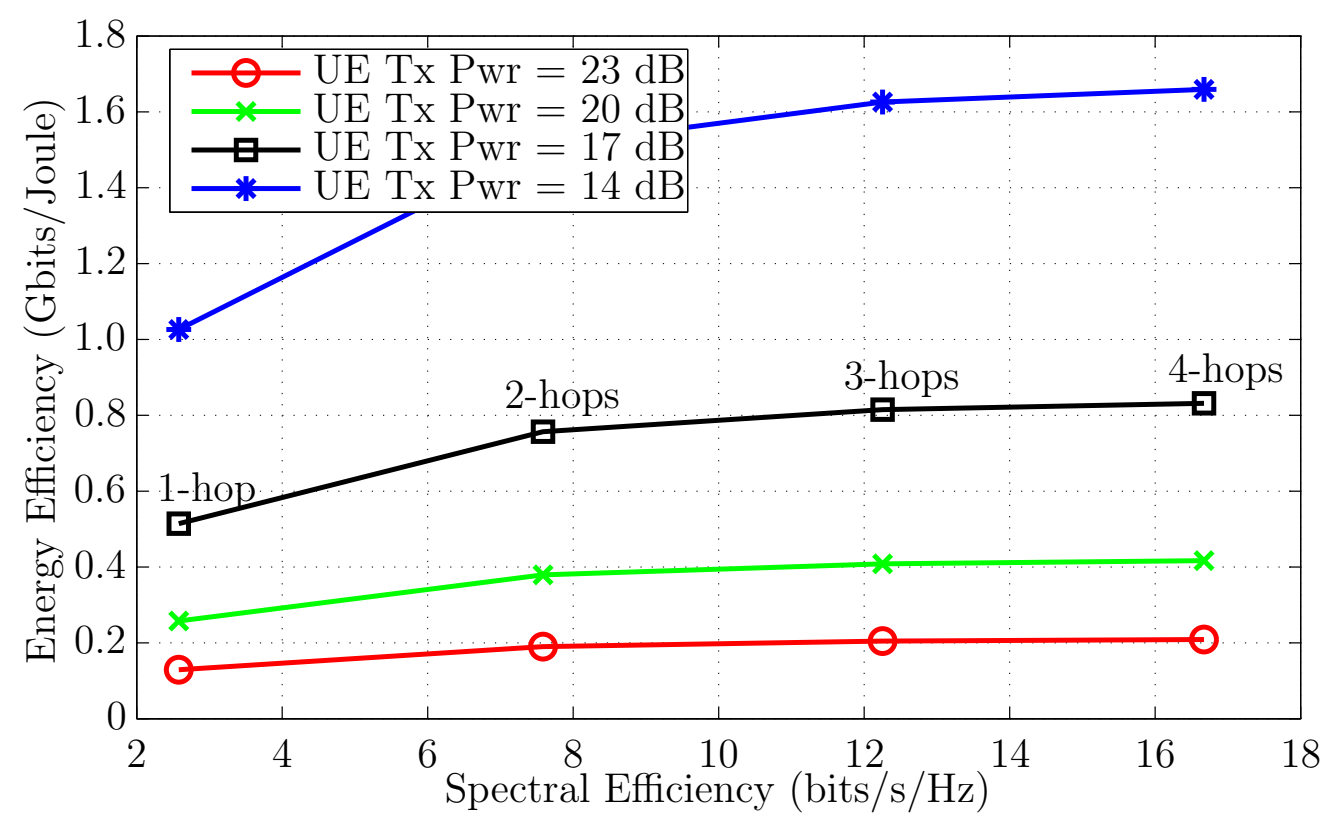

Figure 4.8: Energy efficiency performance vs spectral efficiency while reducing the UE transmission power.

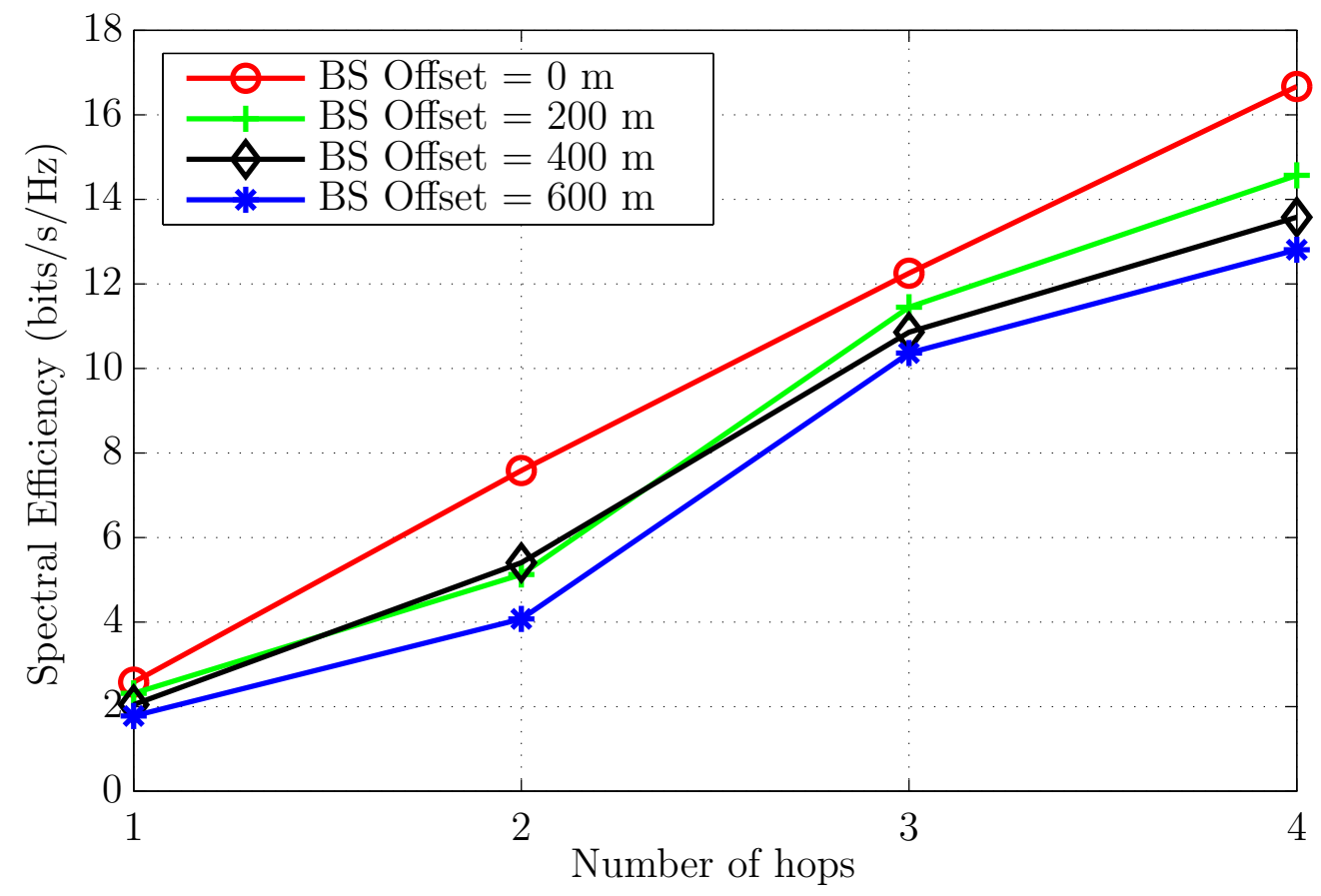

Figure 4.9: Spectral efficiency vs number of hops for different $B S_{2}$ position offsets. 


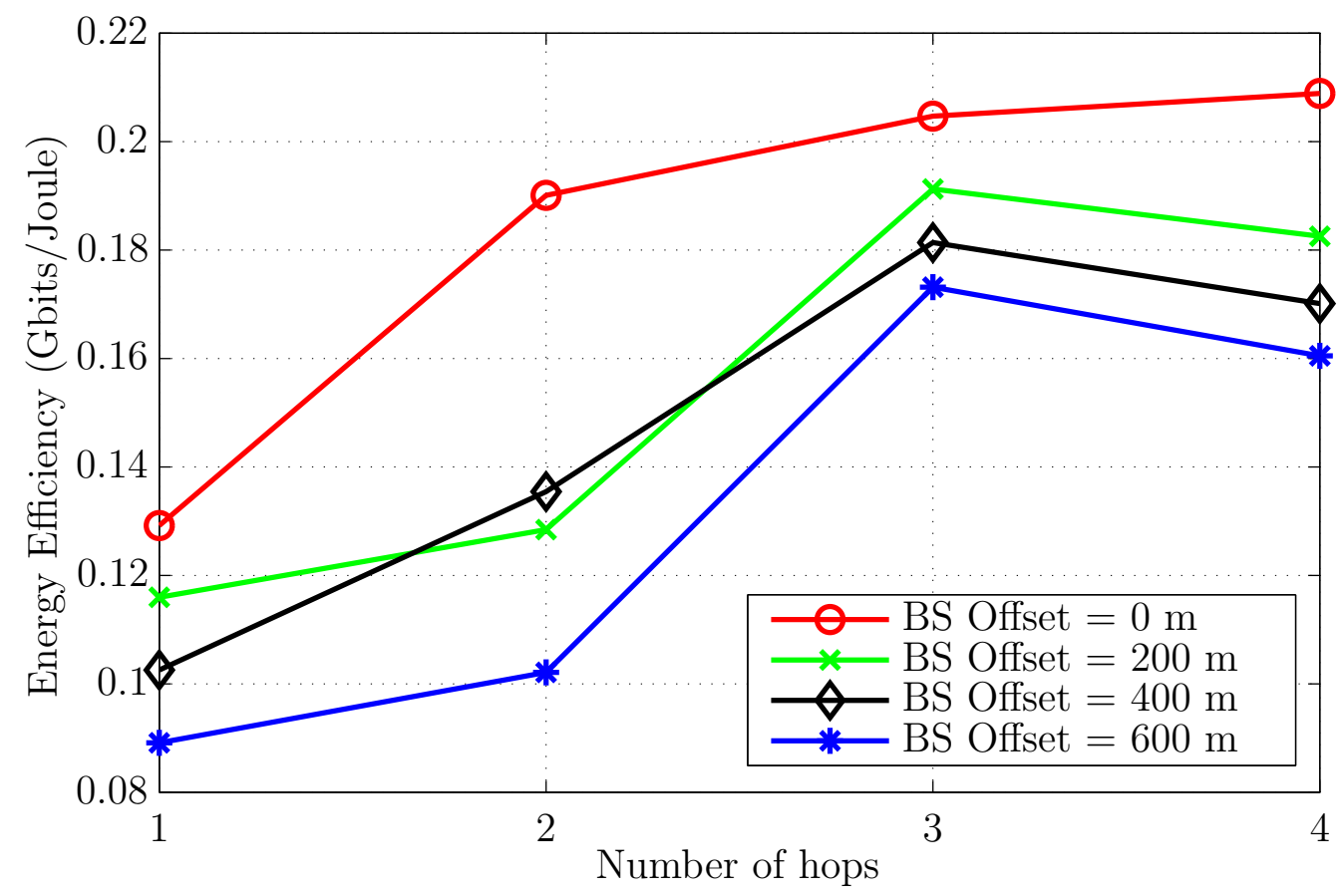

Figure 4.10: Energy efficiency vs number of hops for different $B S_{2}$ position offsets.

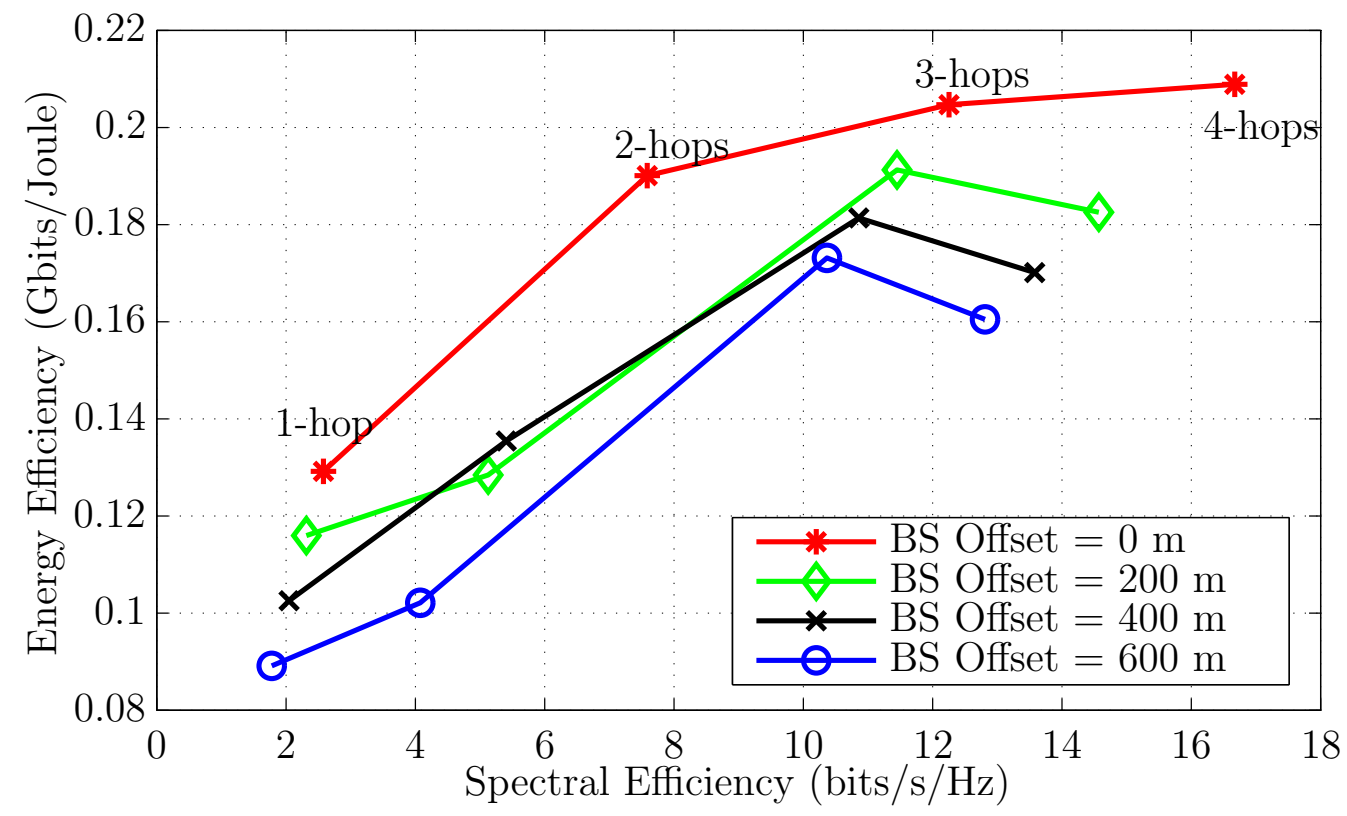

Figure 4.11: Energy efficiency vs spectral efficiency for different $B S_{2}$ position offsets. 


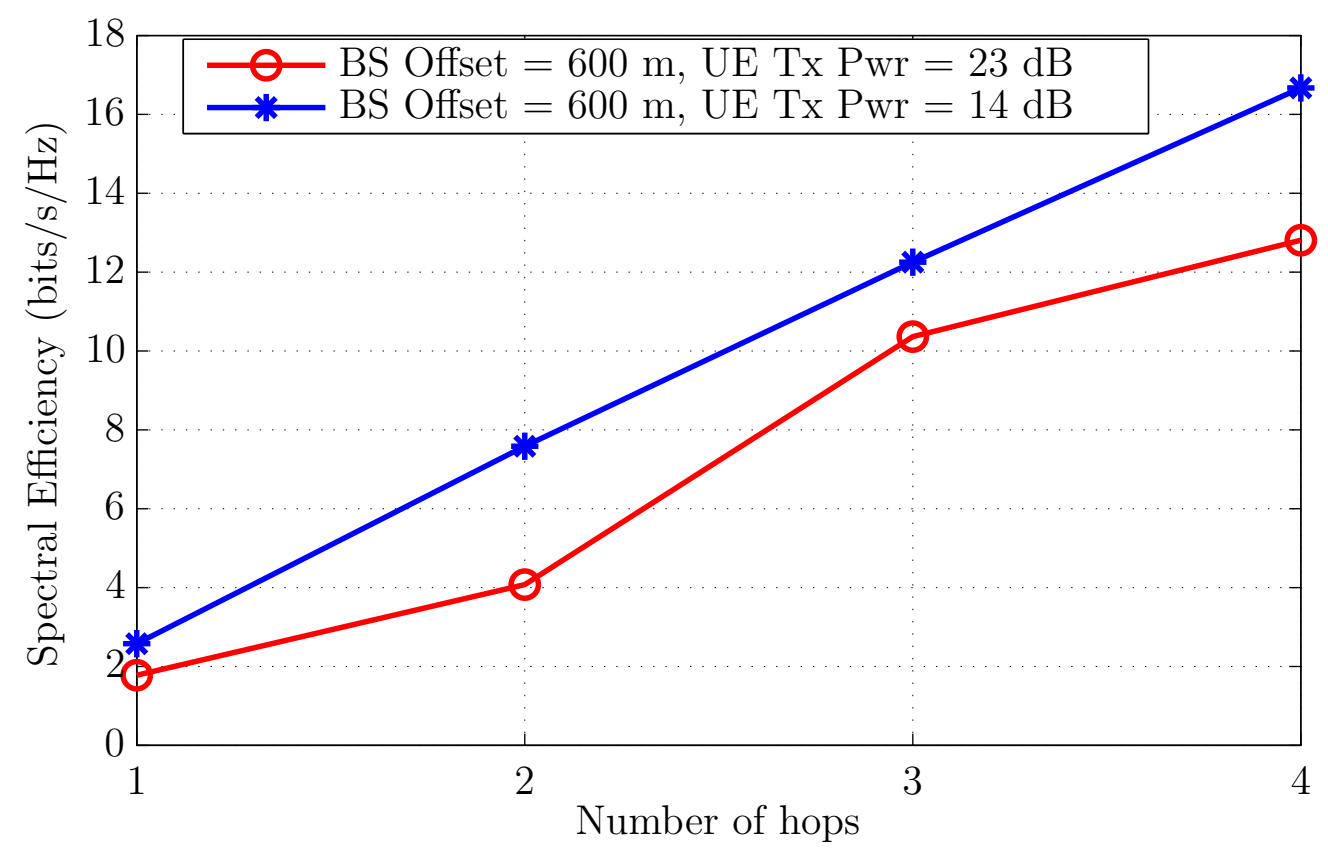

Figure 4.12: Spectral efficiency performance vs number of hops in route 1 with and without power control for high interference scenario.

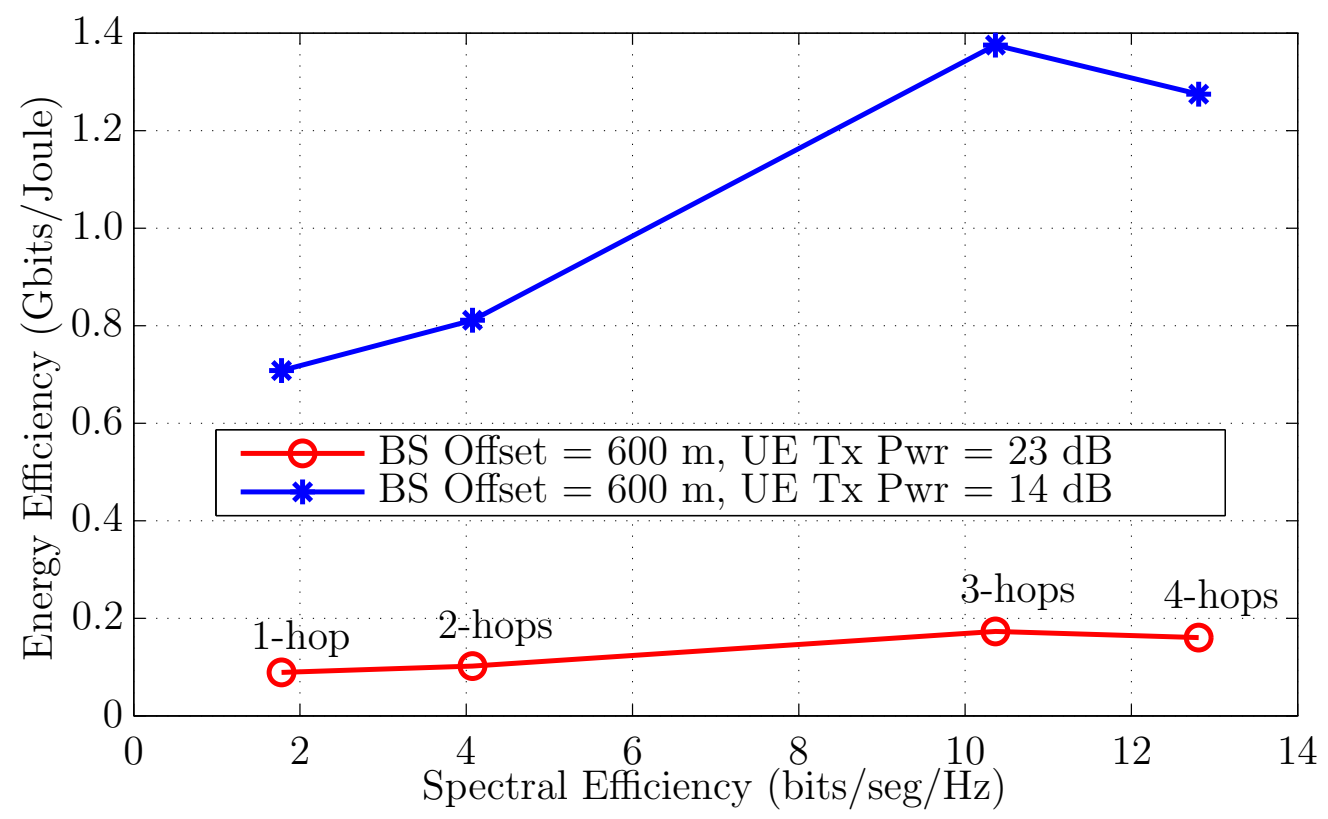

Figure 4.13: Energy efficiency performance vs spectral efficiency for D2D communications in route 1 , with and without power control for high interference scenario. 
Finally, power control is applied to the worst case scenario of interference, that is, the case when route 2 location is $600 \mathrm{~m}$ closer to route 1. Fig. 4.12 and Fig. 4.13 show how D2D communications can benefit from managing UEs transmission power. In this case, when UEs' transmission power is reduced, interference from route 2 decreases, resulting in higher values of SIR and better SE and EE performance. 


\section{CHAPTER 5}

\section{CONCLUDING REMARKS}

There is a growing interest in providing broadband communication services in public safety and emergency communications. In public safety scenarios, communication capabilities need to be provided in very challenging environments where critical infrastructures are often degraded or destroyed by the impact of the catastrophic events. Despite this, first responders should be able to exchange information in a timely manner, securely and reliably.

Based on the requirements and operational context of public safety communications, LTE D2D communications offer remarkable opportunities to provide an evolution path toward broadband capabilities for existing and new public safety networks. Also, D2D represents a viable solution to the problem of partial coverage caused by power outage or damaged MBSs in public safety scenarios.

In this thesis, we studied LTE D2D communications, highlighting those characteristics that define LTE D2D as the technology driver for future public safety communications. Current works in standardization for D2D and ProSe are focused toward that direction.

We also described a 3GPP standard compliant D2D system level simulator including a heterogeneous network deployment based on 3GPP assumptions for public safety. We implemented a received power based PDRID selection algorithm for D2D discovery and proposed an ABS based ICIC approach. In addition, we considered multi-hop based D2D discovery which leads to significant performance improvement based on simulation results.

Finally, the D2D system level simulator is adapted to a public safety partial coverage scenario. Simulation procedures are designed in order to evaluate the per- 
formance of D2D communications with multi-hop in extending the coverage area of active MBSs during emergency situations.

Simulation results demonstrate that by using D2D multi-hop communications, it is possible to communicate far-away-non-covered UEs with active MBSs while achieving excellent performance in capacity, energy efficiency and spectral efficiency, when compared with direct communication between the far-away UEs and the base stations.

D2D communications performance is degraded in the presence of high interference. To improve the performance, multi-hop communications can be used. In particular, larger number of hops result in better EE and SE performance. There is, however, a diminishing return in achievable EE when the number of hops increases.

Power control mechanisms can be used to reduce the impact of D2D interference from neighboring D2D links, in order to obtain a better overall EE/SE performance. The improvements in energy efficiency by setting limits to D2D UEs transmission power led to better use of the energy resources in mobile devices and longer battery life. Additionally, the improvements in spectral efficiency led to better use of spectral resources. Both topics are of critical importance in public safety scenarios.

Future works on this research path should focus on evaluating the performance of different D2D routing techniques. Also, resource allocation, delay and performance evaluation of $\mathrm{EE}$ and $\mathrm{SE}$ can be investigated in more complex scenarios. 


\section{BIBLIOGRAPHY}

[1] 3GPP, "Feasibility study for proximity services (ProSe)," Technical Specification Group SA (TR 22.803), 2012.

[2] M. Gundlach, "Overview of D2D proximity services standarization in 3GPP LTE," in Proc. European Conf. on Networks and Comm., Bologna, Italy, June 2014.

[3] LG Electronics, LG Uplus, Qualcomm, "Discussion on network operation for partial network coverage," 3GPP RAN1 Standard Contribution (R1-132750), Japan, May 2013.

[4] M. Simic, "Feasibility of long term evolution (LTE) as technology for public safety," in Telecomm. Forum (TELFOR), Belgrade, Serbia, Nov. 2012, pp. 158161.

[5] G. B. R. Ferrus, O. Sallent and L. Goratti, "LTE: the technology driver for future public safety communications," IEEE Commun. Mag, pp. 154-161, Oct. 2013.

[6] Department of Homeland Security (The SAFECOM Program), "Statement of requirements for public safety wireless communications and interoperability," Mar. 2006.

[7] T. Doumi, M. Dolan, S. Tatesh, A. Casati, G. Tsirtsis, K. Anchan, and D. Flore, "LTE for public safety networks," IEEE Commun. Mag, pp. 106-112, Feb. 2013.

[8] D. A. G. Baldini, S. Karanasios and F. Vergari, "Survey of wireless communications technologies for public safety," IEEE Commun. Surveys and Tutorials, pp. 619-641, Sept. 2013.

[9] 3GPP, "WID for proximity-based services (normative work) (ProSe)," Work Item Description (SP-120883), 2012.

[10] — - "Group communication system enablers for LTE (GCSE-LTE)," Technical Specification (3GPP TR 23.468), 2013.

[11] _ , "Public safety broadband high power UE for Band 14 for Region 2," Work Item Description (RP-120362), 2012.

[12] K. M. G. L. Goratti, G. Steri and G. Baldini, "Connectivity and security in a D2D communication protocol for public safety applications," in Proc. Int. Symposium on Wireless Commun. Systems. Barcelona, Spain: IEEE, Aug. 2014. 
[13] LG Electronics, LG Uplus, Qualcomm, "Discussion on network operation for partial network coverage," 3GPP RAN1 Standard Contribution (R1-132750), Fukuoja, Japan, May 2013. [Online]. Available: http://www.3gpp.org/ftp/ tsg_ran/wg1_rl1/TSGR1_73/Docs/

[14] A. Asadi, Q. Wang and V. Mancuso, "A survey on device-to-device communication in cellular networks," IEEE Commun. Surveys and Tutorials, Apr. 2014.

[15] K. Doppler, M. Rinne, C. Wijting, C. Ribeiro, and K. Hugl, "Device-to-device communication as an underlay to LTE-advanced networks," IEEE Commun. Mag., vol. 47, no. 12, pp. 42-49, Dec. 2009.

[16] M. Q. G. Fodor, L. Qianxi and N. Brahmi, "Clustering schemes for d2d communications under partial/no network coverage," in Proc. IEEE Broadband Wireless Access Workshop. Atlanta, GA: IEEE, Dec. 2013.

[17] Ch. Tata and M. Kadoch, "Efficient priority access to the shared commercial radio with offloading for public safety in LTE heterogeneous networks," $J$. Computer Networks and Communications, vol. 2014, p. 15, Nov. 2014. [Online]. Available: http://dx.doi.org/10.1155/2014/597425

[18] C. Tata and M. Kadoch, "Multipath routing algorithm for device-to-device communications for public safety over lte heterogeneous networks," in Proc. Int. Conf. Information and Communication Technologies for Disaster Management. Algiers, Algeria: IEEE, Mar. 2014.

[19] H. Yuan, W. Guo and S. Wang, "Emergency route selection for D2D cellular communications during an urban terrorist attack," in Proc. IEEE Int. Conf. on Commun. Sydney, Australia: IEEE, Mar. 2014.

[20] S. Jae Bae, J. Gu and M. Y. Chung, "Two-hop communication scheme for FlashLinQ device-to-device communication system," in Proc. Int. Conf. Information Networking, no. 14. Phuket, Thailand: IEEE, Feb. 2014, pp. 85-90.

[21] G. Rigazzi, F. Chiti, R. Fantacci and C. Carlini, "Multi-hop D2D networking and resource management scheme for M2M communications over LTE-A Systems," in Proc. Wireless Commun. and Mobile Computing Conf. Nicosia, Cyprus: IEEE, Aug. 2014, pp. 973-978.

[22] P. Ren, Q. Du and L. Sun, "Interference-aware routing for hop-count minimization in wireless D2D networks," in Proc. IEEE Int. Workshop on Internet of Things. Xi'an, China: IEEE, Aug. 2013, pp. 65-70. 
[23] D. Astely, E. Dahlman, G. Fodor, S. ParkvalL and J. Sachs, "LTE release 12 and beyond," IEEE Commun. Mag, vol. 51, no. 7, pp. 154-160, 2013.

[24] Alcatel-Lucent and Alcatel-Lucent Shanghai Bell, "Device discovery for D2D proximity services," Chicago, IL, Apr. 2013. [Online]. Available: http://www.3gpp.org/ftp/tsg_ran/WG1_RL1/TSGR1_72b/Docs/

[25] Qualcomm, Inc., "Study on LTE device to device proximity services," 3GPP Work Item Description (RP-122009), Spain, Dec. 2012.

[26] X. Lin, J. G. Andrews, A. Ghosh and R. Ratasuk, "An overview of 3GPP deviceto-device proximity services," Comm. Magazine, IEEE, vol. 51, no. 4, pp. 40-48, Apr. 2014.

[27] M. N. Teharin, M. Uysal and H. Yanicomeroglu, "Device-to-device communication in 5G cellular networks: challenges, solutions, and future directions," Comm. Magazine, IEEE, vol. 52, no. 5, pp. 40-48, May. 2014.

[28] R. Atat, E. Yaacoub, M. Alouini, F. Filali and A. Abu-Dayya, "Delay-sensitive content distribution via peer-to-peer collaboration in public safety vehicular adhoc networks," Ad Hoc Networks, vol. 16, pp. 182 - 196, 2014. [Online]. Available: http://www.sciencedirect.com/science/article/pii/S1570870513002989

[29] D. Feng, L. Lu, Y. Yuan-Wu, G. Y. Li, S. Li and G. Feng, "Device-to-device communications in cellular networks," IEEE Commun. Mag., vol. 52, no. 4, pp. 49-55, Apr. 2014.

[30] K. Doppler, M. Rinne, P. Jannis, C. Ribeiro, and K. Hugl, "Device-to-device communications; functional prospects for LTE-Advanced Networks," in Proc. IEEE Int. Conf. on Comm. Workshops (TELFOR), Dresden, Germany, Jun. 2009, pp. 1-6.

[31] P. Janis, V. Koivunen, C. Ribeiro, J. Korhonen, K. Doppler, and K. Hugl, "Interference-aware resource allocation for device-to-device radio underlaying cellular networks," in Proc. IEEE Vehic. Technol. Conf. (VTC), Apr. 2009, pp. 1-5.

[32] C. Xu, L. Song, Z. Han, Q. Zhao, X. Wang, and B. Jiao, "Interference-aware resource allocation for device-to-device communications as an underlay using sequential second price auction," in Proc. IEEE Int. Conf. Commun. (ICC), June 2012, pp. 445-449. 
[33] S. Mumtaz, K. M. S. Huq, A. Radwan, J. Rodriguez, R. L. Aguiar, "Energy efficient interference-aware resource allocation in LTE-D2D communication," in Proc. Int. Conf on Comm. Sydney, NSW: IEEE, Jun. 2014, pp. 282-287.

[34] M. Simsek, A. Merwaday, N. Correal and I. Guvenc, "Device-to-device discovery based on 3GPP system level simulations," in Proc. IEEE Global Commun. Conf., Workshop on Device-to-Device (D2D) communication with and without infrastructure, Atlanta, GA, Dec. 2013.

[35] L. Babun, M. Simsek and I. Guvenc, "Intercell interference coordination for D2D discovery in LTE-A HetNets," in Proc. IEEE Wireless Commun. and Networking Conf. IEEE WCNC 2014, Apr. 2014.

[36] Intel Corporation, "Discussion on UE-UE channel model for D2D studies," 3GPP Work Item Description (RP-130092), St Julians, Malta, Apr. 2013. [Online]. Available: http://www.3gpp.org/ftp/tsg_ran/wg1_rl1/TSGR1_72b/Docs/

[37] Nokia and Nokia Siemens Networks, "Device to device channel model," Chicago, IL, Apr. 2013. [Online]. Available: http://www.3gpp.org/ftp/tsg_ran/ WG1_RL1/TSGR1_72b/Docs/

[38] F. Junyi, "Device-to-device communications in LTE-advanced network. Networking and internet architecture." Telecom Bretagne, Universite de Bretagne-Sud, 2013.

[39] S. Sesia, I. Toufik, and M. Baker, Eds., LTE - The UMTS Long Term Evolution From Theory to Practice. John Wiley \& Sons, 2011.

[40] MCC Support, "Final report of 3GPP TSG RAN WG1 \#72bis v1.1.0," 3GPP RAN1 Standard Contribution (R1-132646), Chicago, USA, Apr. 2013.

[41] J. Hong, S. Park, H. Kim, S. Choi and K. B. Lee, "Analysis of device-to-device discovery and link setup in LTE networks." in IEEE Int. Symp. Personal Indoor and Mobile Radio Comm. London, UK: IEEE, Sept. 2013.

[42] 3GPP, "Evolved universal terrestrial radio access (E-UTRA); further advancements for E-UTRA physical layer aspects," Technical Report (3GPP TR 36.814), 2010 .

[43] D. Lopez-Perez, I. Guvenc and X. Chu, "Mobility management challenges in 3GPP heterogeneous networks," IEEE Commun. Mag., vol. 50, no. 12, pp. 7078, Dec. 2012. 
[44] 3GPP, "Feasibility study on LTE device to device proximity services - radio aspects," Technical Report (3GPP TR 36.843), 2013.

[45] D. Lopez-Perez, I. Guvenc and X. Chu, "IMobility enhancements for heterogeneous networks through interference coordination," in Proc. IEEE Wireless Comm. and Networking Conf. Workshops. Paris, France: IEEE, April 2012, pp. 69-74.

[46] Winner, "IST-4-027756 WINNER II D1.1.2 V1.2 WINNER II Channel Model," 2008. [Online]. Available: http://www.cept.org/files/1050/documents/ winner2\%20-\%20final\%20report.pdf

[47] 3GPP, "Mobility enhancements in heterogeneous networks," Technical Report (3GPP TR 36.843), 2012.

[48] Alcatel-Lucent and Alcatel-Lucent Shanghai Bell, "LTE D2D dropping and association," 3GPP RAN1 Standard Contribution (R1-131755), Chicago, IL, Apr. 2013.

[49] MCC Support, "Draft Report of 3GPP TSG RAN WG1 \#73 v0.2.0," 3GPP RAN1 Standard Contribution, Japan, May 2013.

[50] J. M. B. da Silva Jr, G. Fodor and T. F. Maciel, "Performance analysis of network-assisted two-hop D2D communications," ArXiv e-prints, Jul. 2014.

[51] L. Goratti, K. M. Gomez, R. Fedrizzi, and T. Rasheed, "A novel device-todevice communication protocol for public safety applications," in Proc. IEEE Global Commun. Conf. Atlanta-USA: IEEE, Dec. 2013.

[52] I. Parvez, M. Jamei, A. Sundararajan and A. I. Sarwat, "RSS based loop-free compass routing protocol for data communication in advanced metering infrastructure (AMI) of smart grid," in Proc. IEEE Int. Symposium on Computational Intelligence, Orlando, FL, Dec. 2014.

[53] W. Lili, R. Hu, Q. Li, and W. Geng, "Energy-efficiency of multi-hop device-todevice communications underlaying cellular networks," in Proc. IEEE Int. Conf. on Comm. Sydney, NSW: IEEE, Jun. 2014.

[54] Z. Zhou, M. Dong, K. Ota, J. Wu, and T. Sato, "Energy efficiency and spectral efficiency tradeoff in device-to-device (D2D) communications," CoRR, vol. abs/1407.1556, 2014. [Online]. Available: http://arxiv.org/abs/1407.1556 